\title{
ON SHORT- AND LONG-TERM VARIATIONS IN QUASI-BAROTROPIC FLOW
}

\author{
A. WIIN-NIELSEN* \\ Joint Numerical Weather Prediction Unit, Air Weather Service Suitland, Md. \\ [Manuscript received July 3, 1961; revised August 17, 1961]
}

ABSTRACT

\begin{abstract}
A comparison is made between the stability properties of the non-divergent and divergent one-parameter models. It is shown that the introduction of divergence in the one-parameter model reduces the rate of growth of unstable disturbances and confines the instability to a more narrow band of wavelengths.

Changes in zonal momentum, momentum transports, and energy conversions between mean flow kinetic energy and eddy kinetic energy are investigated in the linear case, as well as by extended integrations of the spectral form of the prognostic equations allowing only a few wave numbers.

Long-term variations in barotropic flow, where the flow is initially stable, are investigated using the speetral formulation, but allowing only as many wave numbers as are needed to investigate variations in the profile of the zonal wind.
\end{abstract}

\section{INTRODUCTION}

The stability of barotropic flow has been investigated in some detail in earlier studies, notably by Kuo [8] and Eliasen [7]. The extensive use of the barotropic model in operational forecasting has caused a continuing interest in the performance of this model. Certain modifications have been introduced in the forecast equations to alter the forecasts of planetary waves. These semi-empirical corrections are described by Cressman [5] and Wiin-Nielsen [17]. The so-called divergent baratropic model is probably going to be used in operational forecasting for some time to come. It is therefore of interest to study the stability of disturbances in this model and to compare the results with the corresponding results for the non-divergent, one-parameter model. The first part of this paper is concerned with such a study.

There has been recently an increasing interest in the performance of the numerical prediction models when they are integrated over extended periods of time up to several days, weeks, or even months. Thompson [15] has developed a heuristic theory of long-period velocity variations in barotropic flow. It was especially shown from this theory that intense jet stream maxima have a tendency to split into two maxima traveling toward the north and the south, a situation which seems to be characteristic of certain types of atmospheric flow connected with blocking. Lorenz [9] has shown how the barotropic vorticity equation can be reduced to a maximum simplification and has investigated the extended time-integration of such a model. The same approach was used by Bryan [3] to perform experiments with a simple baroclinic model dealing with the different regines

*Present affiliation: National Center for Atmospheric Research, Boulder, Colo. raused by changes of the rotation of the earth and the rate of heating

Saltzman [14] has investigated the non-linear interaction between the zonal flow, large-scale, and smallscale disturbances by an extended integration of the barotropic vorticity equation using a limited number of Fourier components.

The general circulation experiments performed by Phillips [12] deal with an integration of the two-parameter, quasi-geostrophic models over extended periods including adiabatic effects and friction. Extensions of Phillips' work to more realistic models based on the primitive equations and having a greater vertical resolution in addition to more realistic heating and frictional effects are underway in different research groups.

The second part of this paper will describe the variations in the flow of a barotropic fluid on a large time scale. We shall be concerned partly with a flow which is initially unstable, and partly with a flow which is initially stable. Integral quantities and their variation in time will be given because we are not interested in the details of the individual disturbances. The changes in the profile of the zonal wind, the momentum transports, and the energy conversions between the kinetic energies of the mean zonal flow and the disturbances will be reproduced in detail. We shall use a simplification of the barotropic vorticity equation for these purposes of a type similar to Lorenz's minimum form, but shall allow only as many wave components as are needed to describe a zonal wind profile which has at least two maxima.

\section{STABILITY STUDY OF THE DIVERGENT, ONE-PARAMETER MODEL}

The prognostic equation for the divergent, one-param- 
eter model will be given in the form:

$$
\partial \nabla^{2} \psi / \partial t+V \cdot \nabla\left(\nabla^{2} \psi+f\right)=q^{2} \partial \psi / \partial t
$$

where $q^{2}$ is determined by the expression:

$$
q^{2}=\frac{f_{0}^{2}}{\sigma^{2}} \frac{d \sigma}{d p} \frac{d A}{d p} .
$$

The notations have the following meaning: $\psi$ is the stream function, $\mathbf{V}=\mathbf{k} \times \nabla \psi$ the horizontal non-divergent wind, $k$ a vertical unit vector $\nabla^{2}=\partial^{2} / \partial x^{2}+\partial^{2} / \partial y^{2}$ the twodimensional Laplacian, and $f$ the Coriolis parameter. In equation (2.2) $\sigma=-(\alpha / \theta) \partial \theta / \partial p$ is a measure of static stability, $f_{0}$ a standard value of the Coriolis parameter, and $A(p)$ is the empirical function describing the vertical variation of the horizontal wind around the level where the vorticity equation is applied $(500 \mathrm{mb}$.), according to the assumption

$$
\vee(x, y, p, t)=A(p) \mathrm{V}_{5}(x, y, t) .
$$

The subscript 5 has been dropped from the start in equation (2.1). A derivation leading to this particular form of the prognostic equation has been given by WiinNielsen [17].

In the first part of this section we shall consider disturbances on a zonal flow in a region bounded to the north and south by rigid walls. We shall divide any quantity into its zonal mean and deviations from the zonal means. For an arbitrary quantity we write:

where

$$
a=\bar{a}+a^{\prime}
$$

$$
\bar{a}=\frac{1}{L} \int_{0}^{L} a d x .
$$

$I$ is the largest possible wavelength.

When we substitute in (2.1) and neglect second order terms we may derive the following equation:

$$
\nabla^{2} \frac{\partial \psi^{\prime}}{\partial t}+\bar{U} \frac{\partial \zeta^{\prime}}{\partial x}+\left(\beta-\frac{\partial^{2} \bar{u}}{\partial y^{2}}\right) v^{\prime}=q^{2} \frac{\partial \psi^{\prime}}{\partial t} .
$$

We shall consider perturbations in the eddy strean function of the type:

$$
\psi^{\prime}(x, y, t)=\alpha(y) e^{i_{\mu}(x-c t)} .
$$

When we substitute (2.7) into (2.6) we can write the latter equation in the form:

$$
(\bar{u}-c)\left[\frac{d^{2} \alpha}{d y^{2}}-\mu^{2} \alpha\right]+\left[k-\frac{d^{2} \bar{u}}{d y^{2}}+q^{2} c\right] \alpha=0 .
$$

The general eigenvalue problem to find lor which connected values of $u$ and $c$ there exist solutions to equation (2.8), will not be treated in this paper, but we shall restriet ourselves in this first analytical treatment to an initial zonal wind profile of the type

$$
\bar{u}=B\left(1-\cos \frac{2 \pi}{D} y\right)
$$

Interpreting $D$ as the distance between the walls we consider a profile where $u=0$ at both walls and has a value $u=2 B$ for $y=D / 2$, i.e., in the center of the channel.

We shall in the following use an expansion of the function $\alpha=\alpha(y)$ in the form:

$$
\alpha(y)=\sum_{n=1}^{N} \alpha_{n} \sin (n \backslash y)
$$

where $\lambda=\pi / D$. The expression (2.10) for the meridional dependence of the perturbation stream function is chosen in such a way that $\psi^{\prime}$ always disappears at the northern and southern boundaries of the region. The meridional velocity component $v=v^{\prime}$ will therefore also vanish at the boundaries, and the boundary condition corresponding to rigid walls is therefore automatically satisfied.

The expression for $\alpha(y)$ is inserted in equation (2.8) and we obtain after reduction a set of linear homogeneous equations which can be used to determine the possible values of $c$ and the ratios between the values of $\alpha_{n}$. 'Tho' resulting set of equations may be written in the form:

$$
\begin{gathered}
\frac{1}{2}\left(\mu^{2}+\left(n^{2}-4 n\right) \lambda^{2}\right) B \alpha_{n-2}+\left(\left(\mu^{2}+n^{2} \lambda^{2}\right)(c-B)+\beta+q^{2} c\right) \alpha_{n} \\
+\frac{1}{2}\left(\mu^{2}+\left(n^{2}+4 n\right) \lambda^{2}\right) B \alpha_{n+2}=0
\end{gathered}
$$

We notice in the set (2.11) that it divides naturally into two sets corresponding to even and odd values of $n$. It should further be mentioned that we must put a finite value on the maximum number $N$ which $n$ may obtain in order to determine the values of $c$. We shall in this study only be concerned with small and odd values of $n$ and $N$. Larger values of $N$ have been considered by Eliasen [7] but mostly in cases where the $\beta$-effect, and naturally also the effect of divergence, was excluded by assumption.

Restricting ourselves to the most simple case $N=3$ wo obtain the following set of equations from (2.11)

$$
\left.\begin{array}{c}
\left\{\left(\lambda^{2}+\mu^{2}+\ell^{2}\right) c+\beta-\frac{1}{2} B\left(3 \mu^{2}-\lambda^{2}\right)\right\} \alpha_{1} \\
+\frac{1}{2}\left(\mu^{2}+5 \lambda^{2}\right) B \alpha_{3}=0 \\
\frac{1}{2}\left(\mu^{2}-3 \lambda^{2}\right) B \alpha_{1}+\left\{\left(9 \lambda^{2}+\mu^{2}+q^{2}\right) c\right. \\
\left.+\beta-B\left(9 \lambda^{2}+\mu^{2}\right)\right\} \alpha_{3}=0
\end{array}\right\}
$$

$A$ set of equations very similar, but not identical to (2.12) may naturally also be obtained by applying the same technique as Charney [4] in deriving the stability criterion for a two-parameter model with horizontal as well as vertical shear. Instead of the series (2.10) one obtains the equations corresponding to (2.12) by applying 


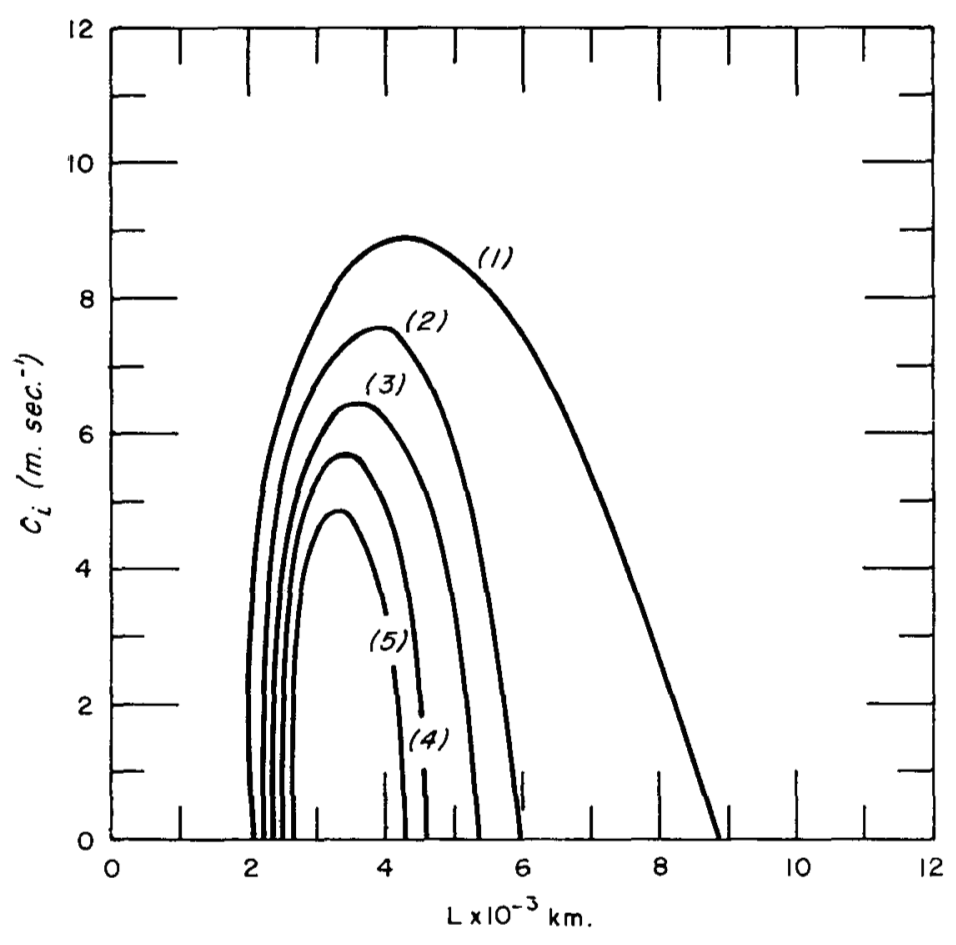

FIgURe 1.-The imaginary part, $c_{i}$, of the solution to equation (2.13) as a function of the zonal wavelength for a maximum meridional wavelength of $4000 \mathrm{~km} ., B=30 \mathrm{~m}$. sec. ${ }^{-1}$ Curve (1): $\beta=0, q^{2}=0 ;(2): \beta=\beta_{45}, q^{2}=0 ;(3): \beta=\beta_{45}, q^{2}=0.5 \times 10^{-12} \mathrm{~m}^{-2}$; (4) : $\beta=\beta_{45}, q^{2}=1.0 \times 10^{-12} \mathrm{~m}^{-2} ;(5): \beta=\beta_{45}, q^{2}=1.5 \times 10^{-12} \mathrm{~m}^{-2}$.

the linearized equation (2.8) in the center of the channel and midway between the center and the wall. Meridional derivatives are replaced by finite differences. It is a matter of convenience whether one or the other procedure is used.

The possible values of $c$ are now determined from the condition that the determinant of the system (2.12) has to vanish in order to have non-trivial solutions. This condition leads to the equation:

$$
\begin{aligned}
& \left(\lambda^{2}+\mu^{2}+q^{2}\right)\left(9 \lambda^{2}+\mu^{2}+q^{2}\right) c^{2}+\left[2 \beta\left(5 \lambda^{2}+\mu^{2}+q^{2}\right)\right. \\
& -1 / 2 B\left(5 \mu^{4}+9 \lambda^{4}+46 \mu^{2} \lambda^{2}+5 \mu^{2} q^{2}\right. \\
& \left.\left.+17 \lambda^{2} q^{2}\right)\right] c+\left[\beta^{2}-1 / 2 \beta B\left(5 \mu^{2}+17 \lambda^{2}\right)\right. \\
& \left.+1 / 4 B^{2}\left(5 \mu^{4}-3 \lambda^{4}+50 \mu^{2} \lambda^{2}\right)\right]=0
\end{aligned}
$$

The frequency equation (2.13) has been solved numerically for a number of cases. These cases fall into different series. For each value of the maximum, meridional wavelength $(2 \pi / \lambda)$ it was decided to vary the zonal wavelength $(L=2 \pi / \mu)$ in each series keeping $q$ constant. The effect of $\beta$ and of divergence was tested by keeping $\beta=0$ and $q=0$ in one series of computations. The results of these computations are given in figures 1,2 , and 3.

The maximum, meridional wavelength in the computations illustrated in figure 1 was $4,000 \mathrm{~km}$. and the max-

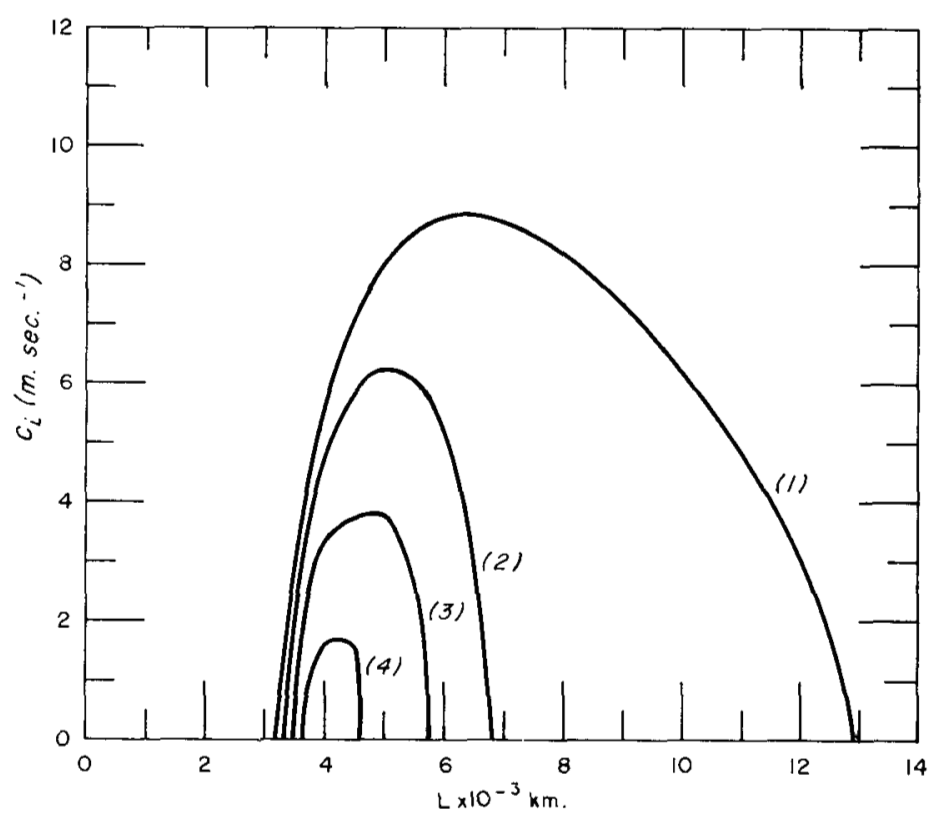

Figure 2.-The imaginary part, $c_{i}$, of the solution to equation (2.13) as a function of the zonal wavelength for a maximum meridional wave length of $6,000 \mathrm{~km}$., $B=30 \mathrm{~m}$. sec. ${ }^{-1}$ The curves (1), (2), (3), and (4) as in figure 1.

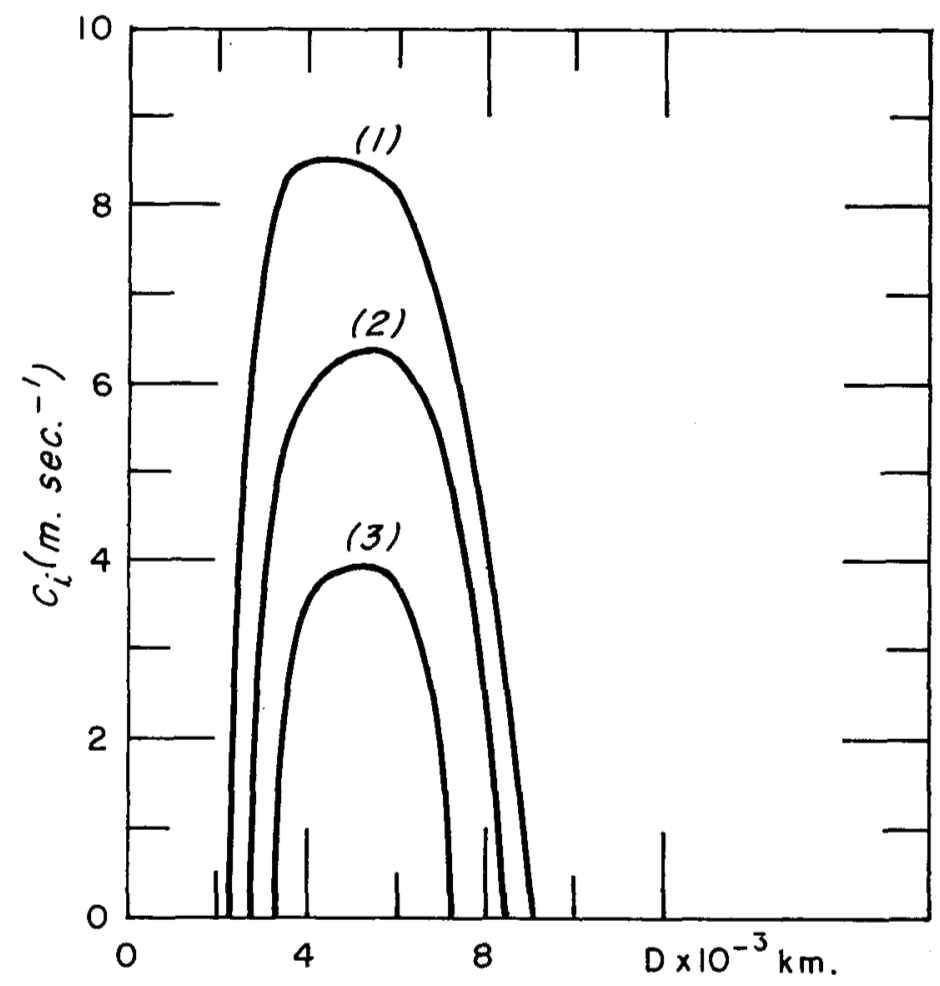

FIGURe 3. - The imaginary part, $c_{i}$, of the solution to equation $(2,13)$ as a function of the meridional wavelength for a fixed zonal wavelength of $5,000 \mathrm{~km}, B=30 \mathrm{~m}$. sec.-1 The curves (1), (2), and (3) as in figure 1.

imum zonal speed in the initial profile $60 \mathrm{~m} . \mathrm{sec}^{-1}(B=$ $30 \mathrm{~m}$. sec. $\left.{ }^{-1}\right)$. This case corresponds to a rather narrow intense jet stream. The abscissa in the figure is the zonal 
wavelength in the unit of thousands of $\mathrm{km}$., while the ordinate is the imaginary part of the wave speed: $c_{i}$. The figure contains five curves corresponding to the cases: (1) $\beta=0, q=0$; (2) $\beta=\beta_{45}, q=0$; (3) $\beta=\beta_{45}, q=0.5 \times 10^{-12} \mathrm{~m}^{-2}$; (4) $\beta=\beta_{45}, q=1.0 \times 10^{-12} \mathrm{~m} .{ }^{-2}$; and (5) $\beta=\beta_{45}, q=1.5 \times 10^{-12}$ $\mathrm{m}^{-2}$

It is seen by comparing the two first cases that the $\beta$ effect has a stabilizing influence on the perturbations in the sense that the magnitude of $c_{i}$ is smaller for $\beta \neq 0$ than in the case $\beta=0$ and also that the band of zonal wavelengths for which instability occurs is more narrow for $\beta \neq 0$. By comparing the first two cases with the last three it is furthermore seen that the effects of $q$, which measures the intensity of the divergence implied by the model, are to further decrease the instability of the waves in the model. The divergent, one-parameter model is therefore more stable than the non-divergent model.

The results are verified by figure 2 , which contains similar calculations with the maximum, meridional wavelength at $6,000 \mathrm{~km}$. The important new information contained in this figure is that the waves are more stable when the meridional wavelength is increased. For $\mathrm{D}=$ $6,000 \mathrm{~km}$. it is seen that all wavelengths are stable in the case of $\beta=\beta_{45}$ and $q=1.5 \times 10^{-12} \mathrm{~m}^{-2}$ and only the waves in a narrow band around $4,000 \mathrm{~km}$. are unstable for $\beta=\beta_{45}$ and $q=1.0 \times 10^{-12} \mathrm{~m} .^{-2}$. Figure 3 contains curves giving the imaginary root as a function of the meridional wavelength for a fixed value of the zonal wavelength, $L=5,000 \mathrm{~km}$.

The information contained in figures 1,2 and 3 may also be expressed in terms of the time, $T$, it would take to double the amplitude of the unstable perturbation. This time can be determined from the formula

$$
T=\frac{\ln 2}{\mu c_{i}}
$$

where $c_{i}$ is the positive, imaginary part of the wave speed. If the wavelength $L=2 \pi / \mu$ is expressed in the unit 1,000 km., $c_{i}$ in m. sec. ${ }^{-1}$, and $T$ in days we obtain

$$
T=1.28 \frac{L}{c_{i}}
$$

The curves in figures 1,2 and 3 indicate doubling times which may be as short as 0.5 day, but more typically are of the order 1-2 days.

The general conclusion from these calculations is therefore that unstable waves are possible even when the $\beta$-effect and the divergence effects are included in the analysis. Both of these effects have a stabilizing influence on the waves in the sense that the waves become more stable for larger values of $\beta$ and $q$ as they do for larger values of the maximum, meridional wavelength.

The general validity of the conclusions which have been reached in this section may be questioned because only the terms corresponding to $n=1$ and $n=3$ in (2.10) have been included in the frequency equation (2.13). If we also included the next odd value, $n=5$, the result would be that (2.13) would be replaced by a cubic equation, while the corresponding equation would be of the fourth degree if also $n=7$ were included. A priori the possibility exists that several amplifying modes will be present in the solution, and that the growth rate of these modes is different from the one found in this section. Such conditions are found in the theory of baroclinic stability (Charney [4], Murray [11]). In the case of barotropic stability, under investigation here, there exists a limited amount of information regarding the higher modes in the paper by Eliasen [7], who has found the roots in the frequency equation up to and including $n=8$. In the case of a symmetric, harmonic velocity profile in the zonal current he finds for $n \leq 8$ that only one unstable mode exists, and that the grow th rate is the same for $3 \leq n \leq 6$, but somewhat smaller for $n=7$ and $n=8$. It is therefore likely that the diagrams in figures 1,2 , and 3 contain the essential features of the stability of the quasi-barotropic flow, although the problem needs further investigation.

\section{SECOND-ORDER MOMENTUM CHANGES}

It is of interest to see what we can say from the solution of this particular perturbation problem about the secondorder effects of the perturbations on the mean flow. Such second-order effects on the mean flow will give the initial changes, but after a while the mean flow will have changed so much that the solution of the perturbation problem no longer applies, which means that the mean flow now starts to alter the structure of the perturbations in a way radically different from our solution. This particular problem will be treated by numerical calculations in the later section.

The second-order changes in the mean flow can be found from the first equation of motion in a form applicable to the model:

$$
\frac{\partial u}{\partial t}+\frac{\partial u u}{\partial x}+\frac{\partial u v}{\partial y}=-\frac{\partial \phi}{\partial x}+f v .
$$

Defining now

$$
\bar{u}=\frac{1}{L} \int_{0}^{L} u d x
$$

and writing

$$
u=\bar{u}+u^{\prime}, v=v^{\prime}, \text { since } \bar{v}=\frac{\partial \psi}{\partial x}=0
$$

we obtain from (2.14)

$$
\frac{\partial \bar{u}}{\partial t}=-\frac{\partial \overline{u^{\prime} v^{\prime}}}{\partial y}
$$

The initial changes in the zonal wind can, therefore, be computed from the convergence of the momentum transport of the perturbations. We must next evaluate this quantity from the solution of the perturbation problem. The general solution for the perturbation 
stream function can be written (see equations (2.7) and (2.10)):

$$
\begin{aligned}
\psi^{\prime}(x, y, t)=\left\{\alpha_{1}{ }^{(1)}\right. & \left.\sin \lambda y+\alpha_{3}{ }^{(1)} \sin 3 \lambda y\right\} e^{i \mu\left(x-r_{1} t\right)} \\
& +\left\{\alpha_{1}{ }^{(2)} \sin \lambda y+\alpha_{3}{ }^{(2)} \sin 3 \lambda y\right\} e^{e_{\mu}\left(x-c_{2} t\right)}
\end{aligned}
$$

where $c_{1}$ and $c_{2}$ are the two solutions of the frequency equation (2.13) and $\alpha_{1}{ }^{(1)}, \alpha_{3}{ }^{(1)}, \alpha_{1}{ }^{(2)}$, and $\alpha_{3}{ }^{(2)}$ are the four (unknown) amplitudes of the wave components in the solution. The amplitudes $(\alpha)$ will, in general, be complex numbers if the wave speed $c$ is complex. Next let the initial perturbation stream function be given by

$$
\psi_{0}{ }^{\prime}(x, y)=\left(A_{1} \sin \lambda y+A_{3} \sin 3 \lambda y\right) e^{i \mu x} .
$$

We have then the following four equations to determine the amplitudes. Two equations are determined by the condition that the expression (3.5) has to agree with (3.6) for $t=0$, giving:

$$
\left.\begin{array}{l}
\alpha_{1}{ }^{(1)}+\alpha_{1}{ }^{(2)}=A_{1} \\
\alpha_{3}{ }^{(1)}+\alpha_{3}{ }^{(2)}=A_{3} .
\end{array}\right\}
$$

The remaining two equations are determined by one of the two equations (2.12), which has to be satisfied for $c=c_{1}$ and $c=c_{2}$. Using the first of the equations (2.12) we obtain

$$
\left.\begin{array}{l}
F\left(c_{1}\right) \alpha_{1}{ }^{(1)}+G{\alpha_{3}}^{(1)}=0 \\
F\left(c_{2}\right) \alpha_{1}{ }^{(2)}+G{\alpha_{3}}^{(2)}=0
\end{array}\right\}
$$

where $F(c)$ and $G$ are defined by the expressions

$$
F(c)=\left(1+\frac{\lambda^{2}}{\mu^{2}}+\frac{q^{2}}{\mu^{2}}\right) c+\frac{\beta}{\mu^{2}}-\frac{1}{2} B\left(3-\frac{\lambda^{2}}{\mu^{2}}\right)
$$

and

$$
G=\frac{1}{2}\left(1+5 \frac{\lambda^{2}}{\mu^{2}}\right) B
$$

The system (3.7), (3.8) becomes eight linear, inhomogeneous equations when the amplitudes $(\alpha)$ and wave velocity $(c)$ are written as complex numbers and the equations are separated into real and imaginary parts. Let the wave speeds be written:

$$
c_{1}=R+i S, \quad c_{2}=R-i S
$$

and the amplitudes:

$$
\left.\begin{array}{l}
\alpha_{1}{ }^{(1)}=X_{1}+i X_{2} \\
\alpha_{1}{ }^{(2)}=Y_{1}+i Y_{2} \\
\alpha_{3}{ }^{(1)}=Z_{1}+i Z_{2} \\
\alpha_{3}{ }^{(2)}=V_{1}+i V_{2}
\end{array}\right\}
$$

It is obvious that the eight quantities: $X_{1}, X_{2}, Y_{1}, Y_{2}, Z_{1}$, $Z_{2}, V_{1}$, and $V_{2}$ can be determined from (3.7) and (3.8) when $R$ and $S$ have been determined from the solution of (2.13). We may therefore consider the real and imaginary parts of the amplitudes of the wave components as known. The final expression for the perturbation stream function may then be written:

$$
\begin{array}{r}
\psi^{\prime}(x, y, t)=e^{\mu S t}\left\{\left(X_{1} \sin \lambda y+Z_{1} \sin 3 \lambda y\right) \cos \mu(x-R t)\right. \\
\left.-\left(X_{2} \sin \lambda y+Z_{2} \sin 3 \lambda y\right) \sin \mu(x-R t)\right\} \\
+e^{-\mu S t}\left\{\left(Y_{1} \sin \lambda y+V_{1} \sin 3 \lambda y\right) \cos \mu(x-R t)\right. \\
\left.-\left(Y_{2} \sin \lambda y+V_{2} \sin 3 \lambda y\right) \sin \mu(x-R t)\right\}
\end{array}
$$

From the knowledge of the expression for the perturbation stream function it is now a straightforward (although tedious) computation to evaluate the convergence of the meridional transport of momentum to be used in (3.4). We find:

$$
\begin{aligned}
\frac{\partial \bar{u}}{\partial t}=2 \mu \lambda^{2}\left[e^{2 \mu s t}\right. & \left(X_{1} Z_{2}-X_{2} Z_{1}\right)+e^{-2 \mu S t}\left(Y_{1} V_{2}-Y_{2} V_{1}\right) \\
& \left.+\left(V_{1} X_{2}-V_{2} X_{1}+Z_{1} Y_{2}-Z_{2} Y_{1}\right)\right] \cdot M(y)
\end{aligned}
$$

where

$$
M(y)=\cos 4 \lambda y-\cos 2 \lambda y .
$$

It can be shown that for our case we will always have

$$
-\left(X_{1} Z_{2}-X_{2} Z_{1}\right)=\left(Y_{1} V_{2}-Y_{2} V_{1}\right)=N^{*}
$$

and

$$
V_{1} X_{2}-V_{2} X_{1}+Z_{1} Y_{2}-Z_{2} Y_{1}=0 .
$$

Using this information we may write (3.14) in the form:

$$
\frac{\partial \bar{u}}{\partial t}=-4 \mu \lambda^{2} N^{*} \sinh (2 \mu S t) \cdot M(y)
$$

or by integration

$$
\bar{u}(y, t)=\bar{u}(y, 0)-2 \frac{\lambda^{2} N^{*}}{S}[\cosh (2 \mu S t)-1] \cdot M(y) .
$$

In the cases which have been treated numerically it has invariably shown up that $N^{*}$ is a positive quantity. This result is in agreement with remarks made by Kuo [8], on the basis of results obtained by Tollmien [16], and we shall show later that we can obtain this result $\left(N^{*}>0\right)$ from physical considerations of the energy conversions between eddy kinetic and mean kinetic energy.

Taking this result for granted at the moment we can then find qualitatively what will happen to the profile of the zonal wind. The function $M(y)$ is plotted in figure 4 as a function of $y / D$. It has a positive maximum in the center of the channel and negative minima on both sides. Since furthermore [cosh $(2 \mu S t)-1]$ is positive for $t>0$ it follows that the last term on the right side in (3.19) has the opposite sign of $M(y)$. We can therefore expect 


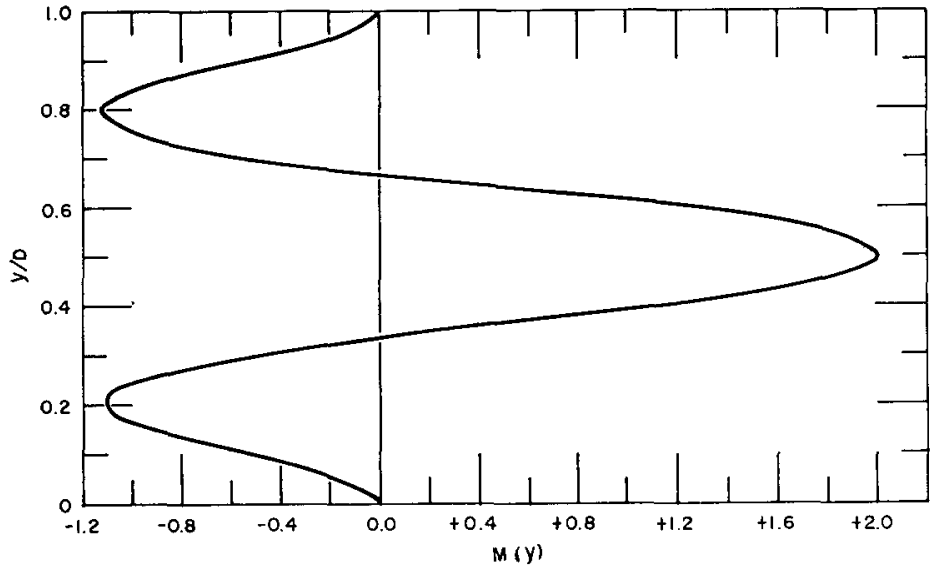

Figure 4.-The function $M(y)$ as defined by equation (3.15).

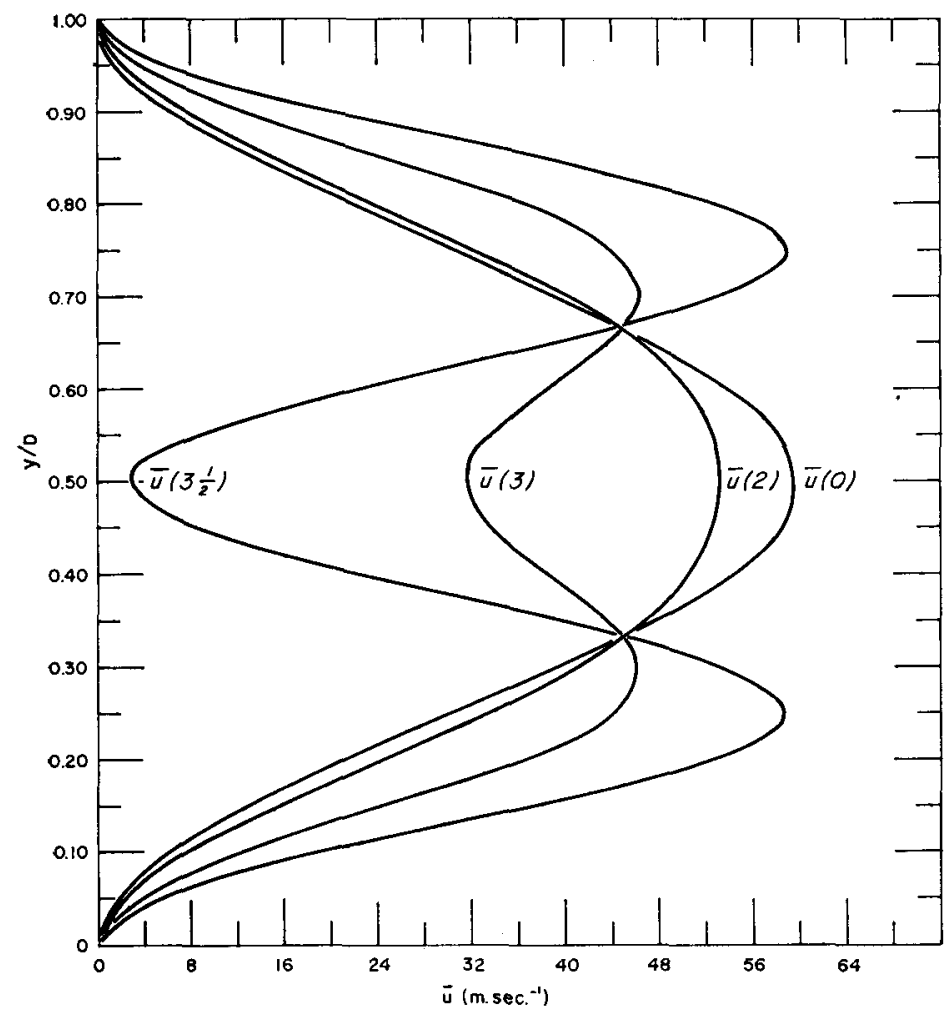

FIGURE 5.-The profile of the zonal wind initially and at 2,3 , and 3.5 days computed from equation (3.19) with $\beta=\beta_{45}, q^{2}=0$, zonal wavelength $5,000 \mathrm{~km}$., width of the channel $3,000 \mathrm{~km}$., $B=30 \mathrm{~m}$. sec. ${ }^{-1}, A_{1}$ corresponds to $5 \mathrm{~m}$. sec. ${ }^{-1}$, and $A_{3}=0$.

that the initial maximum in $\bar{u}(y, 0)$ will be reduced during the first time period after an integration has started while maxima will tend to appear to the north and south of the middle of the channel as time goes on. These results are illustrated in figures 5 and 6 , where we, in figure 5 , have computed the distribution of $u(y, t)$ for $t=0,2,3$, and 3.5 days from equation (3.19). Figure 5 was computed for non-divergent flow $(q=0), \beta=\beta_{45}$, a width of the channel equal to $6,000 \mathrm{~km}$., and a zonal wavelength of $5,000 \mathrm{~km}$. The initial maximum wind speed

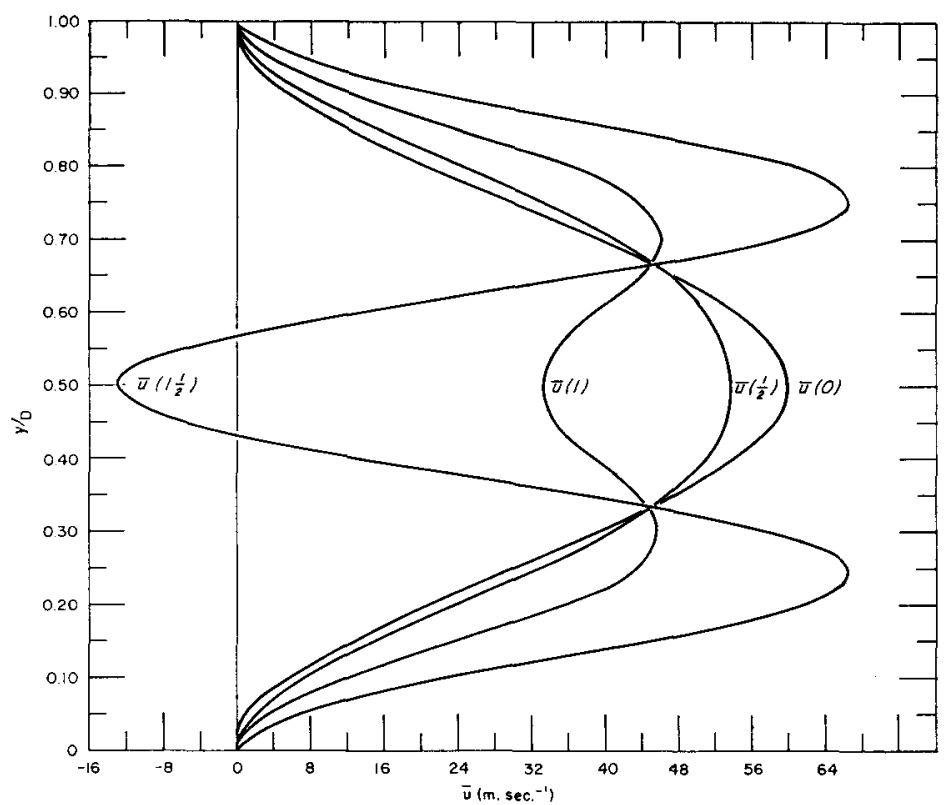

Figure 6 .-The profile of the zonal wind initially and at $0.5,1.0$, and 1.5 days computed from equation (3.19) with parameters as in figure 5 except $A_{3}$ corresponding to $5 \mathrm{~m}$. sec.-1

was $60 \mathrm{~m} . \mathrm{sec}^{-1}$ and $A_{1}$ was chosen corresponding to an initial meridional wind of $5 \mathrm{~m}$. sec. ${ }^{-1}$, while $A_{3}=0$. Figure 5 should be compared with figure 6 where the parameters have the same values except that $A_{3}$ also corresponded to $5 \mathrm{~m}$. sec. ${ }^{-1}$ in the meridional wind component. The distribution of $\bar{u}(y, t)$ is only given for $t=0,0.5,1$, and 1.5 day in figure 6 . It is seen that the presence of the waves on the smaller meridional scale, initially, causes the splitting of the jet stream into the two jet maxima to take place at a faster rate.

Figures 5 and 6 are very similar to those constructed by Thompson [15] from his theory of large-scale turbulence in barotropic flow as they, indeed, should be because the physical model is the same. The changes predicted in the zonal profile in our computation are however, due to the presence of unstable barotropic waves, while Thompson's theory is based upon the interaction between eddies and the mean flow expressed partly in quantities which can be derived from the mean flow and partly in certain statistical properties of the eddies. It will be noted also that the time scale of the changes in the mean zonal flow is the same in the two computations. It takes 2 to 3 days to develop two distinct maxima in the profile of the zonal wind from the single maximum present initially in the center of the channel.

From our calculation we may therefore conclude that if we have barotropic instability in a flow characterized by a single maximum in the zonal wind, it is a dynamical consequence caused by the convergence of the meridional transport of momentum by the eddies that the zonal profile will change to a profile with double maximum. This is done by momentum being transported away from the center of the channel in both directions by the eddies, 
which in turn means that the unstable barotropic disturbances have a tilt of the trough and ridge lines, which goes from southeast toward northwest south of the center and from southwest toward northeast north of the center of the channel. This tilt is opposite to the one generally observed in the atmosphere. This fact points to the importance of barolinic processes in the real atmosphere, but the experiences in barotropic prediction show that processes similar to those described in the present paper definitely occur.

\section{AN EXAMPLE OF BAROTROPIC DEVELOPMENT}

Figure 7 shows a section of the $500-\mathrm{mb}$. analysis from May 15, 1961 at 1200 GMт. The interesting feature on this section of the hemispheric analysis is the fairly straight jet stream from the eastern part of Canada over. Newfoundland toward the southeast over the Atlantic Ocean. Notice in particular that the isotherms are almost parallel to the contours over a large section of the jet stream. The isotachs for the map (not reproduced) show a maximum of about $30 \mathrm{~m}$. sec. ${ }^{-1}$ over Newfoundland with a drop to less than $10 \mathrm{~m}$. sec. ${ }^{-1}$ on both sides in a distance of about $1,000 \mathrm{~km}$.

Figures 8, 9, and 10 show analyses for May 16, 17, and 18 also for 1200 GMT. Between May 15 and 16 a development took place along the jet stream over Newfoundland. The center of the circulation was located approximately at $45^{\circ} \mathrm{N}$., $35^{\circ} \mathrm{W}$. on May 16, $1200 \mathrm{GMT}$. Notice again on figure 8 that we find no regions of strong temperature advection. During the following day the number of closed contours around the center increased, while the Low drifted slowly toward the southeast with a position at $43^{\circ} \mathrm{N} ., 30^{\circ} \mathrm{W}$. on May 17,1200 Gмт. The Low remained almost stationary during the next 24-hour period with a slight tendency toward filling.

The forecasts made with the $500-\mathrm{mb}$. operational model from the initial data at May 15, 1961 at $1200 \mathrm{GMT}$ are shown in figures 11,12 , and 13 . The 24-hour forecast (fig. 11) shows that the barotropic model forecasted the development of a closed circulation. The center located at approximately $50^{\circ} \mathrm{N} ., 35^{\circ} \mathrm{W}$, is too far to the north. However, the barotropic development continued in the forecast between 24 and 48 hours and the circulation is centered at $45^{\circ} \mathrm{N}, 29^{\circ} \mathrm{W}$. in the 48 -hour forecast. The 72-hour forecast shows a further increase in the number of closed contours, but the center has now moved too far toward the east.

The initial jet stream has been divided into two branches on the observed maps and on the forecasts. Toward the end of the period we find a rather broad current over the eastern part of North America. This current divides into two branches, one going toward the northeast over the southern part of Greenland, the other bending toward the southeast and continuing on the southern side of the Low, which was developed in the Atlantic.

The development of the flow pattern in the example

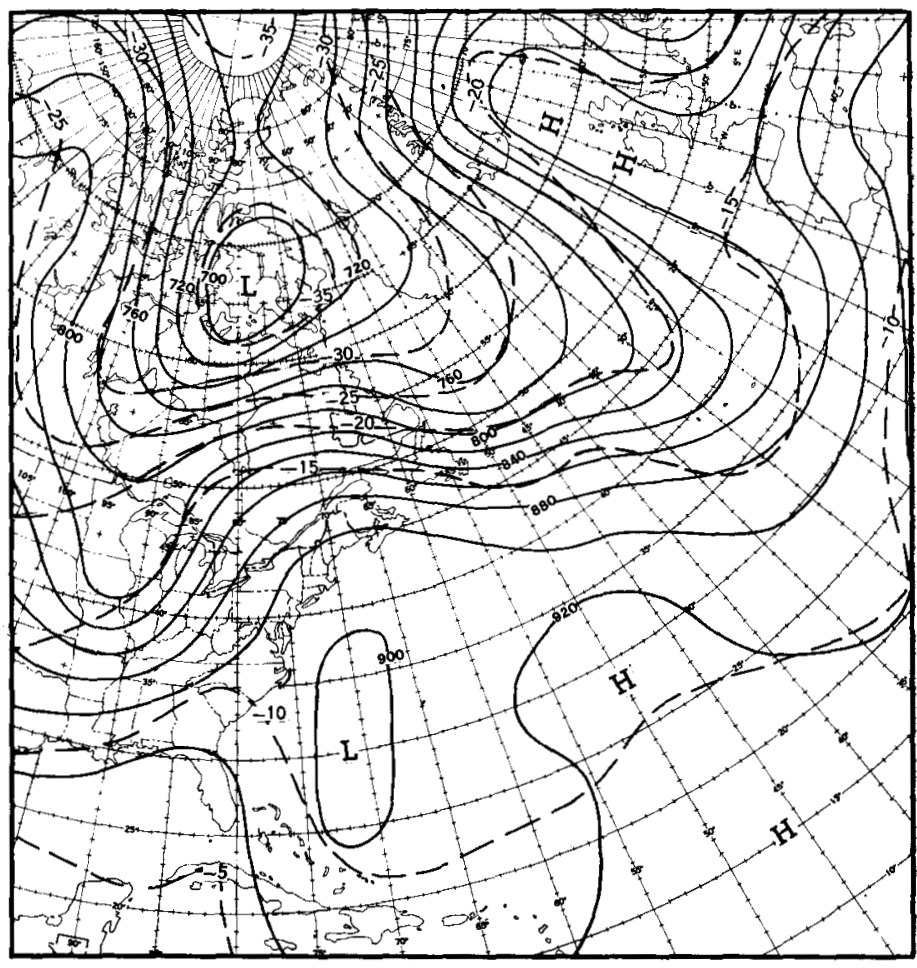

Figcre 7.-500-mb. analysis for May 15, 1961, 1200 GMT. Solid lines are contours and dashed lines isotherms.

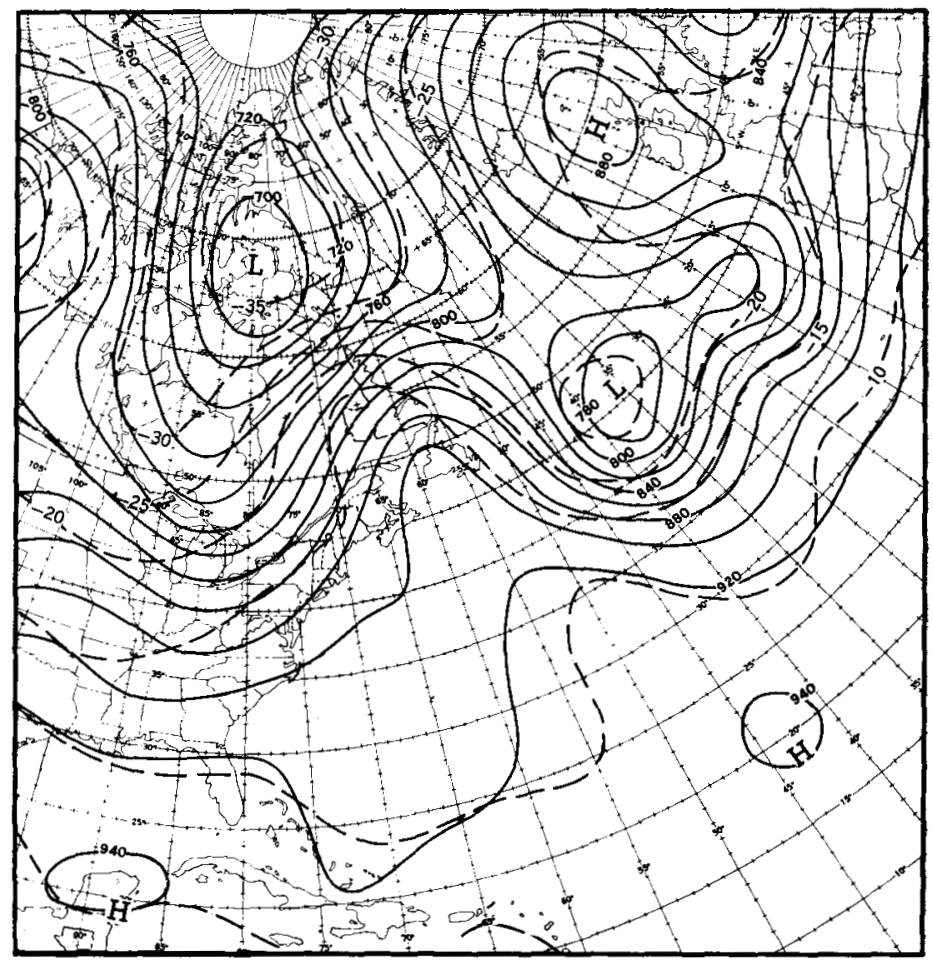

Frgure 8.-500-mb. analysis for May 16, 1961, 1200 GMT.

shows therefore a great similarity to those predicted by the linear theory. This type of barotropic development is observed frequently in barotropic forecasting and results usually in cold, cut-off cyclones. However, it is not the 


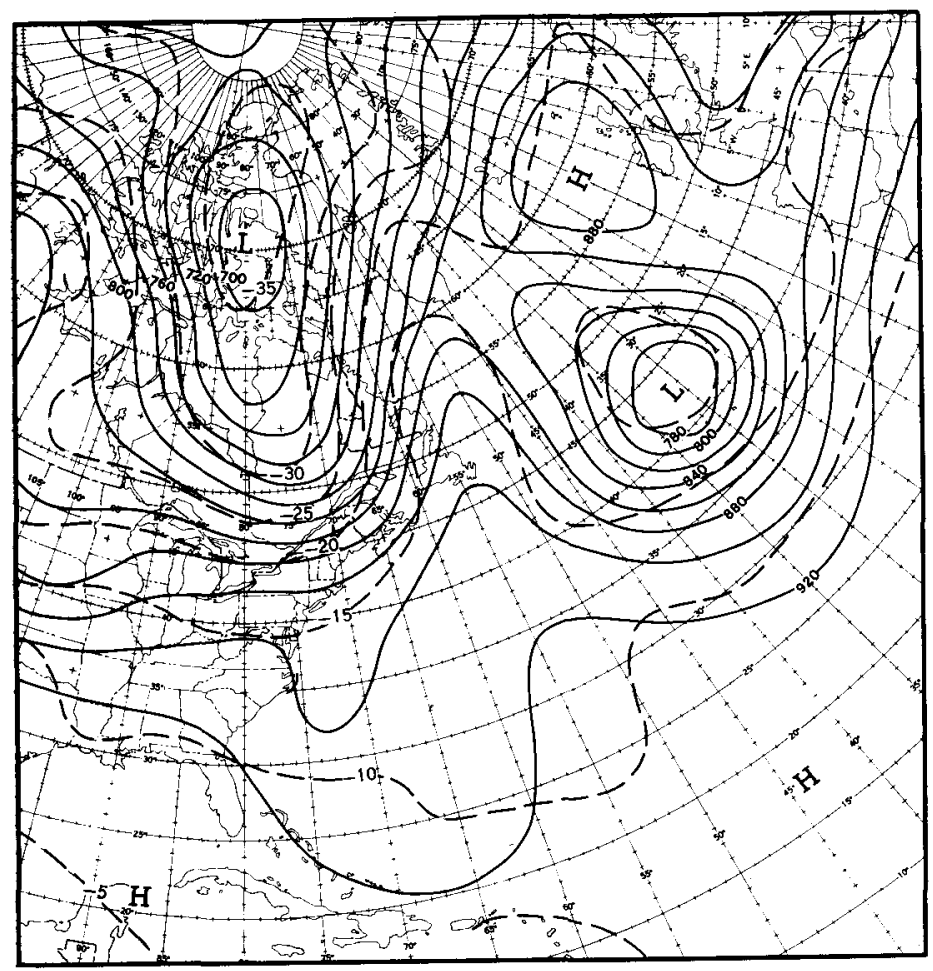

Figure_9.-500-mb. analysis for May 17, 1961, $1200 \mathrm{GmT}$.

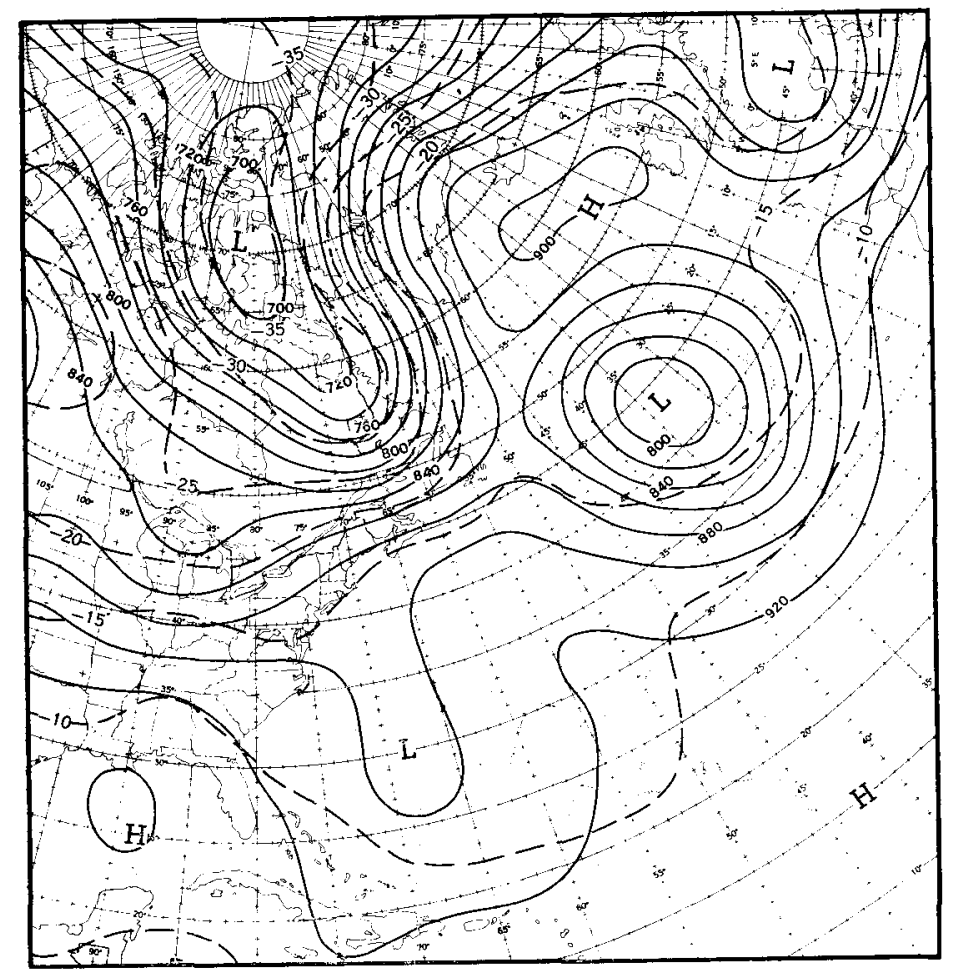

FIGURe 10,-500-mb. analysis for May 18, 1961, 1200 GMT.

most frequent type of development in the troposphere. The baroclinic developments associated with well developed divergence fields are the more common.

It is interesting to note that Murray [11] in a recent investigation of baroclinic stability of stratospheric flow,

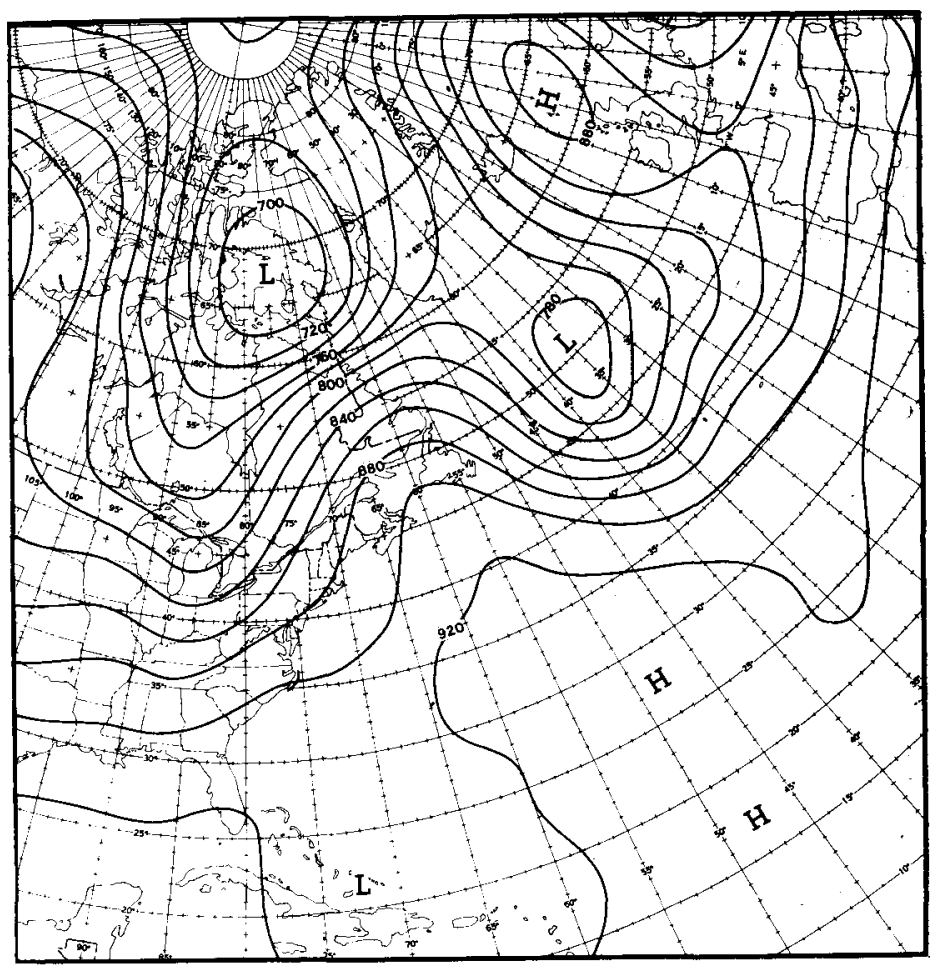

FIGURE 11.-24-hour one-parameter forecast starting from May 15, 1961, 1200 GMT.

especially the breakdown of the polar night jet located close to the $25-\mathrm{mb}$. surface, indicates that the polar night jet is baroclinically and inertially stable. He suggests, therefore, that barotropic instability of the type discussed here is responsible for the breakdown of the polar night jet. This result is contradicted by the conclusions reached by Boville [2] who states that "baroclinic wave development, fulfilling instability criteria and energy conversions of the Fleagle type appear to have been identified down to wave number four." The difference in the results of the two (independent) studies can probably be ascribed to the different models which were adopted, but it is beyond the scope of this paper to resolve the question of the stability of the polar night jet.

\section{ENERGY CONVERSIONS}

We shall next turn our attention to the energy conversion between mean zonal kinetic energy and eddy kinetic energy, the only energy conversion which is possible in the model, if $q=0$; i.e., no divergence. The prediction equation is in this case

$$
\partial \zeta / \partial t+V \cdot \nabla \zeta+\beta v=0
$$

When we divide each quantity into its zonal mean value and deviation from the zonal mean value and introduce these expressions into (5.1) we obtain the following equation for the average flow:

$$
\partial \bar{\zeta} / \partial t+\overline{V^{\prime} \cdot \bar{\nabla} \zeta^{\prime}}=0
$$




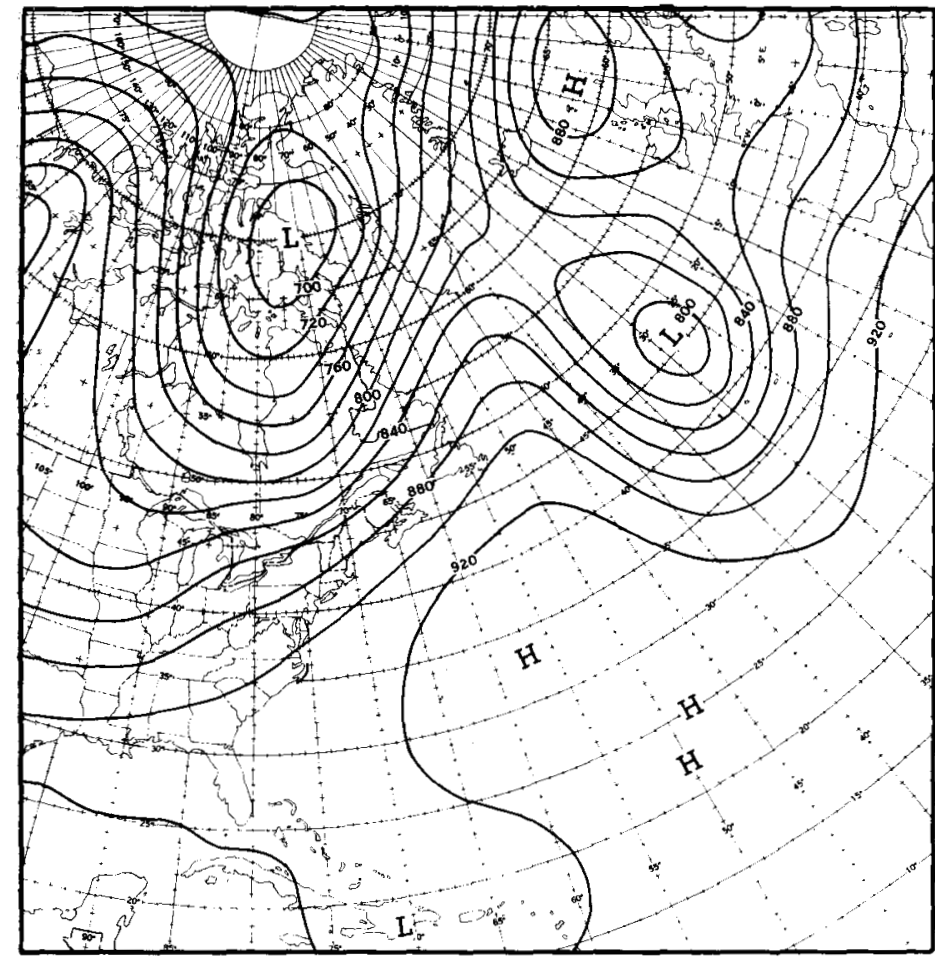

FIGLRE 12.-48-hour one-parameter forecast starting from May $15,1961,1200 \mathrm{GMT}$.

This equation may also be written in the form:

$$
\partial \bar{\zeta} / \partial t=\partial^{2}\left(\overline{u^{\prime} v^{\prime}}\right) / \partial y^{2}
$$

because

$$
\overline{\mathbf{V}^{\prime} \cdot \nabla \zeta^{\prime}}=\nabla \cdot\left(\overline{\zeta^{\prime} \mathbf{V}^{\prime}}\right)=\frac{\partial}{\partial y}\left(\overline{\zeta^{\prime} v^{\prime}}\right)=-\frac{\partial}{\partial y}\left(\overline{v^{\prime} \frac{\partial u^{\prime}}{\partial y}}\right)
$$

but due to the non-divergence of the eddy-flow; i.e.,

$$
\frac{\partial u^{\prime}}{\partial x}+\frac{\partial v^{\prime}}{\partial y}=0
$$

we obtain

$$
-\frac{\partial}{\partial y}\left(\overline{\left.v^{\prime} \frac{\partial u^{\prime}}{\partial y}\right)}\right)=-\frac{\partial^{2} \overline{u^{\prime} v^{\prime}}}{\partial y^{2}}+\frac{\partial}{\partial y}\left(\overline{u^{\prime} \frac{\partial v^{\prime}}{\partial y}}\right) .
$$

The last term is, however, zero, because

$$
\frac{\partial}{\partial y}\left(\overline{u^{\prime} \frac{\partial v^{\prime}}{\partial y}}\right)=-\frac{\partial}{\partial y}\left(\overline{\left(u^{\prime} \frac{\partial u^{\prime}}{\partial x}\right.}\right)=0 .
$$

The mean kinetic energy per unit mass and unit area may in this model be written in the form:

$$
\bar{K}=\frac{1}{S} \int_{S} \frac{1}{2} \bar{u}^{2} d S=\frac{1}{2 D} \int_{0}^{D}\left(\frac{\partial \bar{\psi}}{\partial y}\right)^{2} d y
$$

from which it follows that

$$
\frac{d \bar{K}}{d t}=\frac{1}{D} \int_{0}^{D} \frac{\partial \bar{\psi}}{\partial y} \frac{\partial}{\partial y}\left(\frac{\partial \bar{\psi}}{\partial t}\right) d y=-\frac{1}{D} \int_{0}^{D} \bar{\psi} \frac{\partial \bar{\xi}}{\partial t} d y,
$$

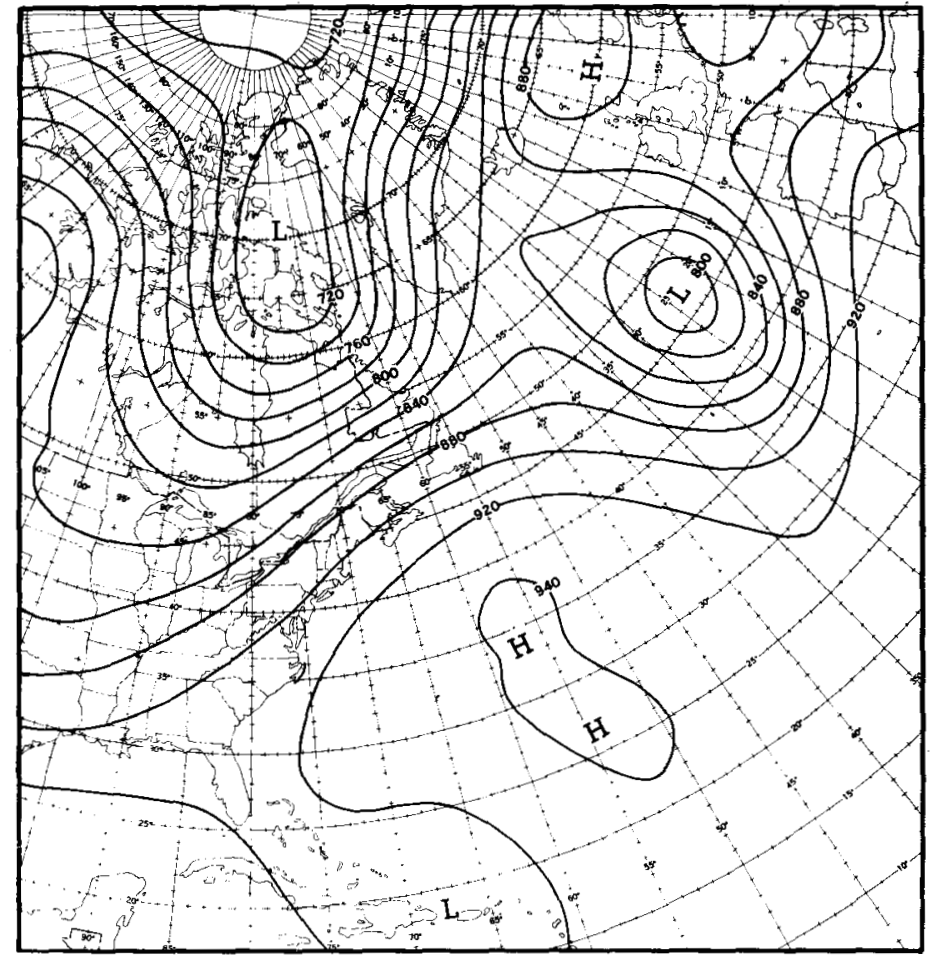

FigURE 13.-72-hour one-parameter forecast starting from May 15, 1961, $1200 \mathrm{G}$ MT.

because $\partial^{2} \bar{\psi} / \partial y \partial t=-\partial \bar{u} / \partial t=0$ at both boundaries at all times. It is seen from (5.9) that we obtain the change in kinetic energy by multiplying (5.3) by $\bar{\psi}$ and then integrating over the width of the channel. This procedure leads to

$$
\frac{d \bar{K}}{d t}=-\frac{1}{D} \int_{0}^{D} \bar{\psi} \frac{\partial^{2} \overline{u^{\prime} v^{\prime}}}{\partial y^{2}} d y=-\frac{1}{D} \int_{0}^{D} \bar{u} \frac{\partial \overline{u^{\prime} v^{\prime}}}{\partial y} d y
$$

The change in time of the eddy kinetic energy may be found from a similar method which results in

$$
\frac{d K^{\prime}}{d t}=\frac{1}{D} \int_{0}^{D} \bar{u} \frac{\partial \overline{u^{\prime} v^{\prime}}}{\partial y} d y
$$

It is then natural to define the following expression as the energy conversion between eddy kinetic energy and mean kinetic energy:

$$
\left\{K^{\prime} \cdot \bar{K}\right\}=-\frac{1}{D} \int_{0}^{D} \bar{u} \frac{\partial \overline{u^{\prime} v^{\prime}}}{\partial y} d y
$$

The general expression for energy conversion in isobaric; non-divergent flow shows that if the zonal wind is positively correlated with the convergence of momentum transport there is a conversion from eddy kinetic energy. to the mean flow kinetic energy, while negative correlations between the two quantities give the opposite conversion.

The energy conversion (5.12) will now be evaluated for 
the amplifying barotropic wave according to the analysis made earlier in this paper. Restricting ourselves to this part of the solution we find that the convergence of momentum transport is given by (3.14)

$$
-\frac{\partial \overline{u^{\prime} v^{\prime}}}{\partial y}=2 \mu \lambda^{2}\left(X_{1} Z_{2}-X_{2} Z_{1}\right) e^{2 \mu S t} \cdot M(y) .
$$

When we substitute this expression together with the expression (2.9) for $\bar{u}$ in (5.12) we find after evaluation of the integral

$$
\left\{K^{\prime} \cdot \bar{K}\right\}=\mu \lambda^{2} B e^{2 \mu S t}\left(X_{1} Z_{2}-X_{2} Z_{1}\right)=-\mu \lambda^{2} B e^{2 \mu S t} N^{*} .
$$

The last expression gives us the sign of the quantity $N^{*}$ which was introduced in (3.16). The eddy kinetic energy of the unstable barotropic wave can only increase as a result of the conversion of energy from the kinetic energy of the mean flow. The energy conversion $\left\{K^{\prime} \cdot \bar{K}\right\}$ is therefore negative, which means that $N^{*}=-\left(X_{1} Z_{2}\right.$ $-X_{2} Z_{1}$ ) must be positive. The sign of $N^{*}$ was of vital importance for the discussion of the changes in the zonal wind profile carried out earlier in the paper.

It should be noted that the expression (5.14), on which we have based the conclusion that $N^{*}$ is positive, is evaluated without regard to the second-order changes in the zonal momentum. The expression (5.14), applies therefore only for a short interval of time initially, a fact which does not destroy the argument. The importance of the higher-order changes in the energy conversion and the question of when the non-linear effects become significant will be investigated at the end of section 6 .

It is obvious that the linearized treatment of the barotropic problem can describe the development of the profile of the zonal wind and perturbations only up to the time when there is considerable interaction between the two parts of the motion. This is seen directly in the expressions which we have derived for the change in the zonal wind (3.18) and the energy conversion (5.14) which will predict changes which tend toward infinity with increasing time.

From energy considerations it is equally obvious that the source of energy for the perturbation, i.e., the zonal available kinetic energy, is finite, and that the growth of the pertubation therefore must stop after a while, and the energy conversion must change sign. The non-linear interactions which are necessary for such processes can only be incorporated by integrating a more complete form of the prediction equation. Lorenz [9] has recently shown a simplification of the non-linear vorticity which enables us to retain the non-linear interaction by restricting the fields to a few wave components. An integration of this type will be described in the next section.

\section{EXTENDED TIME INTEGRATIONS OF BAROTROPIC FLOW}

The extended time integration of the barotropic equation was carried out for two reasons. It was de- sirable to investigate the validity of the results obtained from the linear analysis, and the changes in the zonal profile, the momentum transport, and the energy conversions could be investigated as a function of time in a more general case. The integration can be considered as an extension of Lorenz's [9] original integration, which was made with equations which were simplified to a maximum extent. Guided by the results which we have obtained during the linear analysis we shall formulate a system which is general enough to allow double maxima in the zonal wind profile, but simple enough to give a very efficient computation.

The form of the expression for the mean zonal wind (3.19) in the linear case suggests that an expression of the form :

$\bar{u}(y, t)=B(t)+C(t)-B(t) \cos (2 \lambda y)-C(t) \cos 4 \lambda y$

may be used with advantage in the numerical experiment. The family of curves described by (6.1) for different values of $B$ and $C$ can vary between strong westerlies in the center of the channel with easterlies near the walls and, on the other extreme, strong westerlies near the walls with easterlies in the center of the channel. The predicted values of $B$ and $C$ from given initial values will therefore show the changes in certain types of zonal wind profiles predicted from the barotropic equations. The stream function corresponding to the mean zonal wind $(6.1)$ is:

$$
\bar{\psi}(y, t)=D(B+C)\left[1-\frac{y}{D}\right]+\frac{B}{2 \lambda} \sin (2 \lambda y)+\frac{C}{4 \lambda} \sin (4 \lambda y)
$$

where $D$ is the width of the channel and the constant of integration has been determined in such a way that $\bar{\psi}(D, t)=0$.

The solution (3.13) for the perturbation stream function suggests, on the other hand, a prescribed expression for this quantity of the following form:

$$
\begin{aligned}
\psi^{\prime}(x, y, t)= & \frac{E_{1}(t)}{k} \sin \lambda y \sin k x+\frac{E_{3}(t)}{k} \sin 3 \lambda y \sin k x \\
& +\frac{F_{1}(t)}{k} \sin \lambda y \cos k x+\frac{F_{3}(t)}{k} \sin 3 \lambda y \cos k x
\end{aligned}
$$

which allows one wave number $(k)$ in the zonal direction and two wave numbers ( $\lambda$ and $3 \lambda$ ) in the meridional direction. The complete stream function is then given by the expression

$$
\psi(x, y, t)=\vec{\psi}(y, t)+\psi^{\prime}(x, y, t)
$$

Now (6.4) can be substituted in the vorticity equation

$$
\frac{\partial \nabla^{2} \psi}{\partial t}=J\left(\nabla^{2} \psi, \psi\right)-\beta \frac{\partial \psi}{\partial x}
$$

in which $\beta=d f / d y$ is considered to be a constant, and we 
can by the technique developed by Lorenz [9] transform (6.5) into six forecast equations for the six amplitudes: $B, C, E_{1}, E_{3}, F_{1}$, and $F_{3}$, which are functions of time only. With the notations:

$$
Q=\frac{\lambda^{2}}{k^{2}}, R=\frac{\beta}{k^{2}}
$$

the six forecast equations for the amplitudes are:

$$
\left.\begin{array}{l}
\frac{d B}{d t}=2 k Q\left[E_{1} F_{3}-E_{3} F_{1}\right] \\
\frac{d C}{d t}=-2 k Q\left[E_{1} F_{3}-E_{3} F_{1}\right] \\
\frac{d E_{1}}{d t}=k\left[C F_{1}-\frac{Q-3}{2(Q+1)} B F_{1}-\frac{5 Q+1}{2(Q+1)} B F_{3}-\frac{7 Q-1}{2(Q+1)} C F_{3}-\frac{R}{Q+1} F_{1}\right] \\
\frac{d E_{3}}{d t}=k\left[(B+C) F_{3}+\frac{3 Q-1}{2(9 Q+1)} B F_{1}-\frac{15 Q-1}{2(9 Q+1)} C F_{1}-\frac{R}{9 Q+1} F_{3}\right] \\
\frac{d F_{1}}{d t}=-k\left[C E_{1}-\frac{Q-3}{2(Q+1)} B E_{1}-\frac{5 Q+1}{2(Q+1)} B E_{3}-\frac{7 Q-1}{2(Q+1)} C E_{3}-\frac{R}{Q+1} E_{1}\right] \\
\frac{d F_{3}}{d t}=-k\left[(B+C) E_{3}+\frac{3 Q-1}{2(9 Q+1)} B E_{1}-\frac{15 Q-1}{2(9 Q+1)} C E_{1}-\frac{R}{9 Q+1} E_{3}\right]
\end{array}\right\}
$$

We notice from the first two expressions in (6.7) that

$$
B+C=M_{0}=\text { const. }
$$

This result could have been derived a priori because it simply expresses the fact that total zonal momentum of the system is conserved.

It has been shown by Lorenz [9] that the simplified barotropic equations (6.7) conserve the kinetic energy and the square of the vorticity when these quantities are integrated over the region. It should be noted that the kinetic energy and the square of the vorticity are evaluated as the sum of the contributions from the components which are included in the forecasts.

The kinetic energy per unit area is in our case evaluated from the expression.

$$
K=\frac{1}{L D} \int_{0}^{L} \int_{0}^{D} \frac{1}{2} \nabla \psi \cdot \nabla \psi d x d y=-\frac{1}{2} \cdot \frac{1}{L D} \int_{0}^{L} \int_{0}^{D} \psi \nabla^{2} \psi d x d y
$$

We introduce the expressions $(6.4),(6.3)$, and (6.2) in (6.9) and obtain after evaluation of the integrals

$$
\begin{aligned}
K=\frac{1}{2}(B+C)^{2}+\frac{1}{4}\left(B^{2}+C^{2}\right)+ & \frac{1}{8} \frac{\lambda^{2}+K^{2}}{K^{2}}\left(E_{1}{ }^{2}+F_{1}{ }^{2}\right) \\
& +\frac{1}{8} \frac{9 \lambda^{2}+K^{2}}{K^{2}}\left(E_{3}{ }^{2}+F_{3}{ }^{2}\right) .
\end{aligned}
$$

A direct proof that the kinetic energy is conserved can be obtained by differentiating $(6.10)$ with respect to time and substituting from the system (6.7). This computa- 'tion was carried out as a check on the derivation of the system (6.7)

Let us further define the mean square of the vorticity by the formula

$$
V=\frac{1}{L D} \int_{0}^{L} \int_{0}^{D}\left(\nabla^{2} \psi\right)^{2} d x d y
$$

Substituting again from (6.4) in (6.11) we obtain the following expression for $\mathrm{V}$ :

$$
\begin{aligned}
V=2 \lambda^{2} B^{2}+8 \lambda^{2} C^{2}+\frac{1}{4} \frac{\left(\lambda^{2}+k^{2}\right)^{2}}{k^{2}}\left(E_{1}{ }^{2}+F_{1}^{2}\right) \\
+\frac{1}{4} \frac{\left(9 \lambda^{2}+k^{2}\right)^{2}}{k^{2}}\left(E_{3}{ }^{2}+F_{3}{ }^{2}\right) .
\end{aligned}
$$

Again we can of course obtain a direct proof that $V$ as expressed in (6.12) is indeed conserved. The two conserved quantities, $K$ and $V$, can be computed in each time step during a numerical integration of the system (6.7). If they are conserved during the integration, or at least show no trend for systematic increase, decrease, or violent fluctuations, we have a good check on the behavior of the numerical integration especially with respect to truncation errors. Such a check was introduced in the numerical integration to be described later in this section.

It is one of the purposes of the numerical integration to investigate the energy conversion between mean kinetic energy and eddy kinetic energy during an extended period of integration. We need therefore to evaluate the formula for this energy conversion in our simplified model. This is 
accomplished by a substitution of (6.4) in (5.12) from which we obtain

$$
\left\{K^{\prime} \cdot \bar{K}\right\}=\frac{\lambda^{2}}{\bar{k}}(B-C) \cdot\left(E_{1} F_{3}-E_{3} F_{1}\right) .
$$

The energy conversion (6.13) was computed in each time step during the numerical integration.

We shall next return to the fact that the total momentum of the fluid is conserved as shown in (6.8). This conservative property puts a constraint on the amount of zonal kinetic energy which can be released to the perturbations, as shown by Platzman and Baer [13]. We may, in other words, consider the total zonal kinetic energy as composed of two parts: the available zonal kinetic energy and the unavailable energy. It is easy to evaluate the two quantities in the simple model used in our experiments. The zonal part of the kinetic energy is, as can be seen from $(6.10)$,

$$
E_{z}=\frac{1}{2}(B+C)^{2}+\frac{1}{4}\left(B^{2}+C^{2}\right) .
$$

We can combine (6.14) with (6.8) and rewrite (6.14) in the form:

$$
E_{z}=\frac{3}{4} M_{0}{ }^{2}+\frac{1}{2} B\left(B-M_{0}\right)
$$

from which it is seen that

$$
\delta E_{z}=\left(B-\frac{1}{2} M_{0}\right) \delta B .
$$

$E_{z}$ is therefore at a minimum if $B=1 / 2 M_{0}$ in which case we also have $C=1 / 2 M_{0}$. In our case there exists therefore a particular profile of the zonal wind, described by (6.1), for which the zonal kinetic energy is at a minimum, namely the one for which $B=C$. The minimum value of the zonal kinetic energy is evaluated from (6.14) with $B=C=1 / 2 M_{0}$ and is

$$
E_{2, \text { min. }}=\frac{5}{8} M_{0}^{2}
$$

This is the unavailable part of the zonal kinetic energy. Only $3 / 8$ of the initial momentum squared is therefore available for conversion to eddy kinetic energy. Subtracting (6.17) from (6.15) we can evaluate the available zonal kinetic energy. It becomes

$$
E_{z, a v .}=\frac{1}{8} M_{0}^{2}-\frac{1}{2} B C=\frac{1}{8}(B-C)^{2} .
$$

We notice here that if $B=C=\frac{1}{2} M_{0}$ we get no available, zonal, kinetic energy. It should further be mentioned that very large values of zonal, available energy are obtained in the case that $M_{0}=0$, which means that $B=-C$, in which case $E_{2, a v}=\frac{1}{2} B^{2}$. We shall illustrate later by

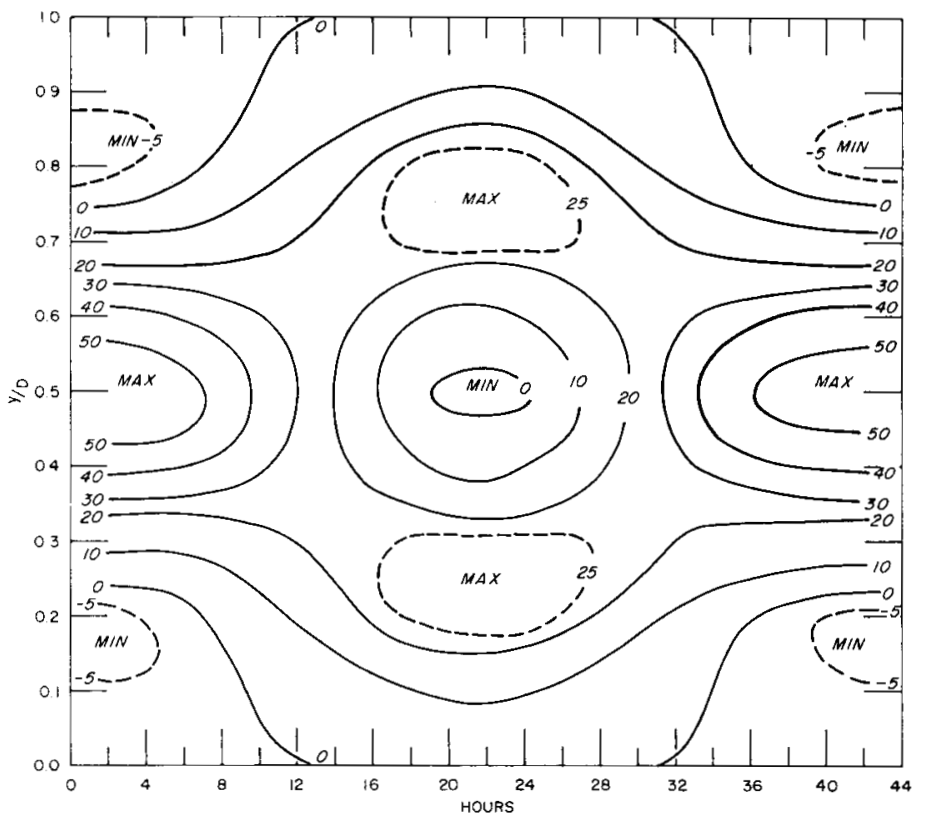

Figlre 14.-Case 1. The zonal velocity as a function of time and meridional distance over one period. Initial parameters: $B_{a}=$ $30 \mathrm{~m}$. sec. ${ }^{-1}, C_{o}=-15 \mathrm{~m}$. sec. ${ }^{-1}, E_{10}=25 \mathrm{~m}$. sec. ${ }^{-1}, E_{80}=F_{10}=$ $F_{30}=0, \beta=\beta_{45}$, width $3,000 \mathrm{~km}$., zonal wavelength $5,000 \mathrm{~km}$.

the results of the actual calculations that very large changes in the zonal flow take place in this case.

Numerical integrations of the system (6.7) have been carried out in a number of cases. In the following we shall describe some of the more interesting results. The integration was performed using central finite differences in time except initially, where one uncentered step was taken. The time step has been 1 hour in all the calculations. We shall treat four different cases. The calculations were in all cases carried out to 24 days (576 time steps), but since all the computations turned out to be periodical we shall usually illustrate only a shorter time period. The finite difference system in time was tested by computing the kinetic energy per unit area and the square of the vorticity per unit area. These quantities showed only small fluctuations during the complete integration, indicating that the finite difference system used in these calculations is good enough even for extended integrations with the present system. A short description of the results of four integrations follows.

Case 1: As the first example we have selected a case where the initial flow pattern is barotropically unstable. The zonal wavelength was taken to be $5,000 \mathrm{~km}$. and the width of the channel $3,000 \mathrm{~km}$., corresponding to a maximum meridional wavelength of $6,000 \mathrm{~km}$. The quantities $B$ and $C$ were selected initially at $30 \mathrm{~m}$. sec. ${ }^{-1}$ and -15 m. sec. ${ }^{-1}$, respectively, giving a zonal wind profile with a single maximum in the center of the channel of $60 \mathrm{~m}$. sec. ${ }^{-1}$ The profile has also weak easterlies close to the walls with a maximum easterly wind of about $7 \mathrm{~m}$. $\mathrm{sec}^{-1}$ The initial disturbance was defined by putting $E_{1}=25 \mathrm{~m} . \mathrm{sec}^{-1}$, 


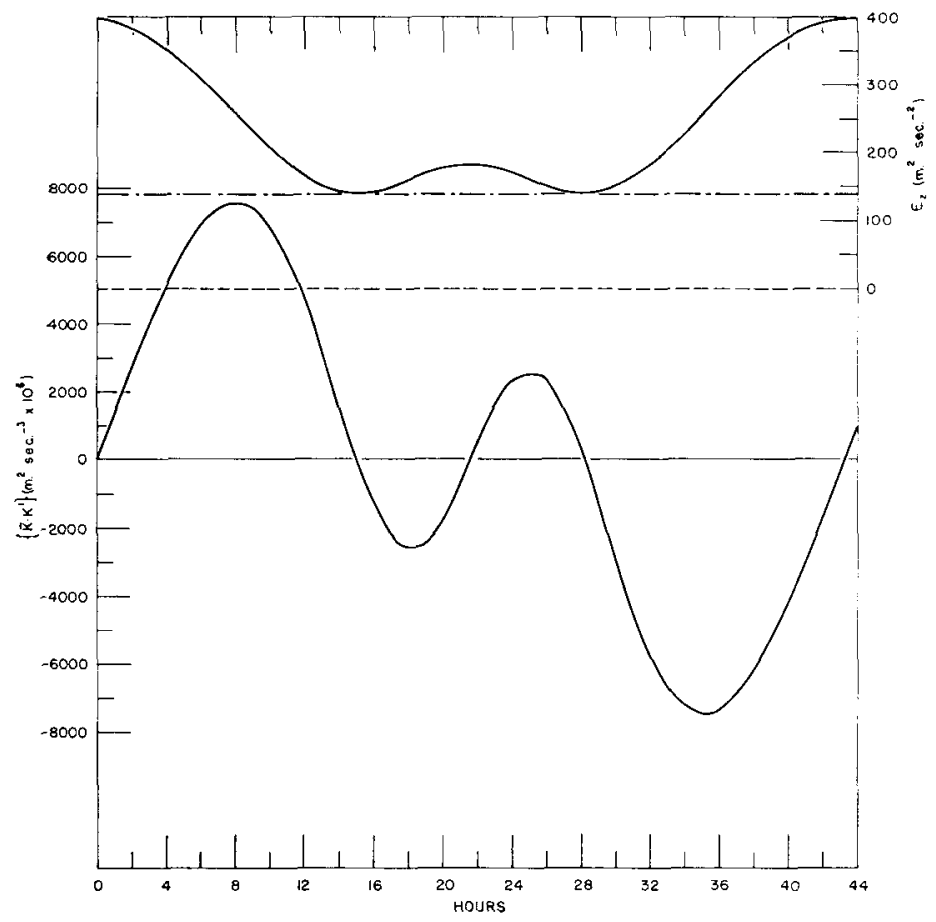

Figure 15.-Case 1. The zonal kinetic energy as a function of time over one period (upper curve, right scale). The energy conversion between zonal and eddy kinetic energy as a function of time over one period (lower curve, left scale).

while $E_{3}=F_{1}=F_{3}=0$ initially. The disturbance defined by these values has a south-north direction of the trough and ridge lines and has, therefore, initially no transport of momentum.

The changes which take place in the zonal winds during the forecast are illustrated in figure 14, where the zonal wind profile is shown as a function of time in hours. The forecast turns out to be periodic with a period close to 44 hours. We have therefore only shown one period. The initial maximum in the zonal wind in the center of the channel breaks down and is after about 22 hours replaced by weak easterlies of $2-3 \mathrm{~m}$. sec.-1 At the same time two maxima of westerly zonal winds form to the north and the south of the center of the channel with speeds of $28-29 \mathrm{~m}$. sec. ${ }^{-1}$ After 44 hours we are back to the initial zonal wind profile. The forecast predicts therefore an oscillation in the zonal wind profile varying between a single and a double maximum in the jet stream.

The processes which determine this oscillation are described in figure 15, in which the lower curve shows the energy conversion between zonal kinetic energy and eddy kinetic energy. During the first hours of the forecast the energy is converted from the zonal flow to the eddies. This conversion reaches a maximum after about 8 hours, but remains positive up to about 15 hours, when the sign of the conversion changes for a few hours and becomes zero shortly before 22 hours. The upper curve shows the variation with respect to time of the kinetic energy contained in the zonal flow. In agree-

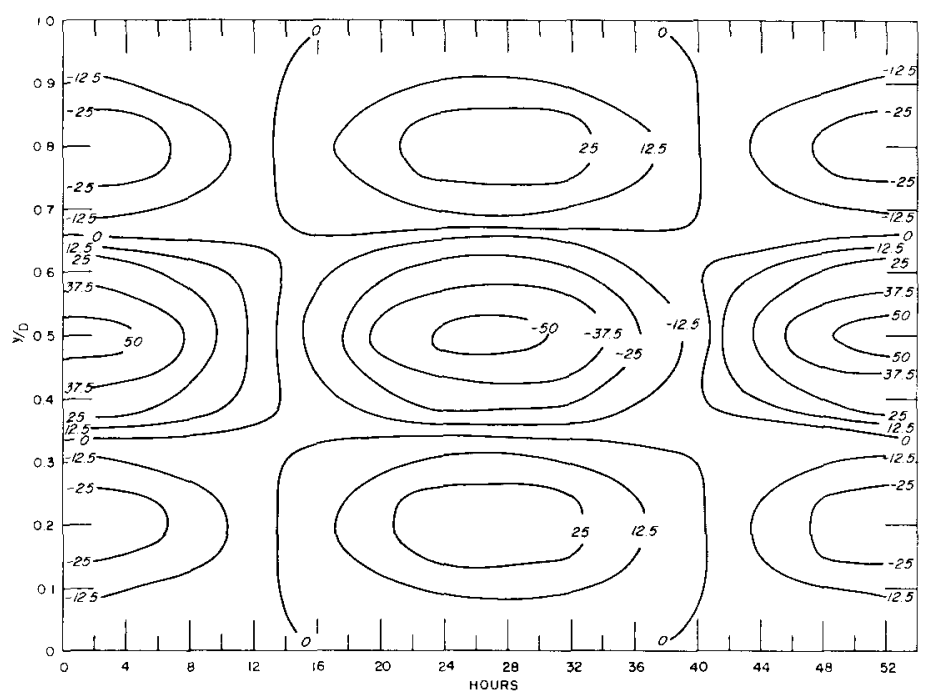

Figure 16. - Case 2. Zonal velocity as a function of time and meridional distance over one period. Parameters as in case 1 (fig. 14) except $C_{o}=30 \mathrm{~m}$. sec. ${ }^{-1}$.

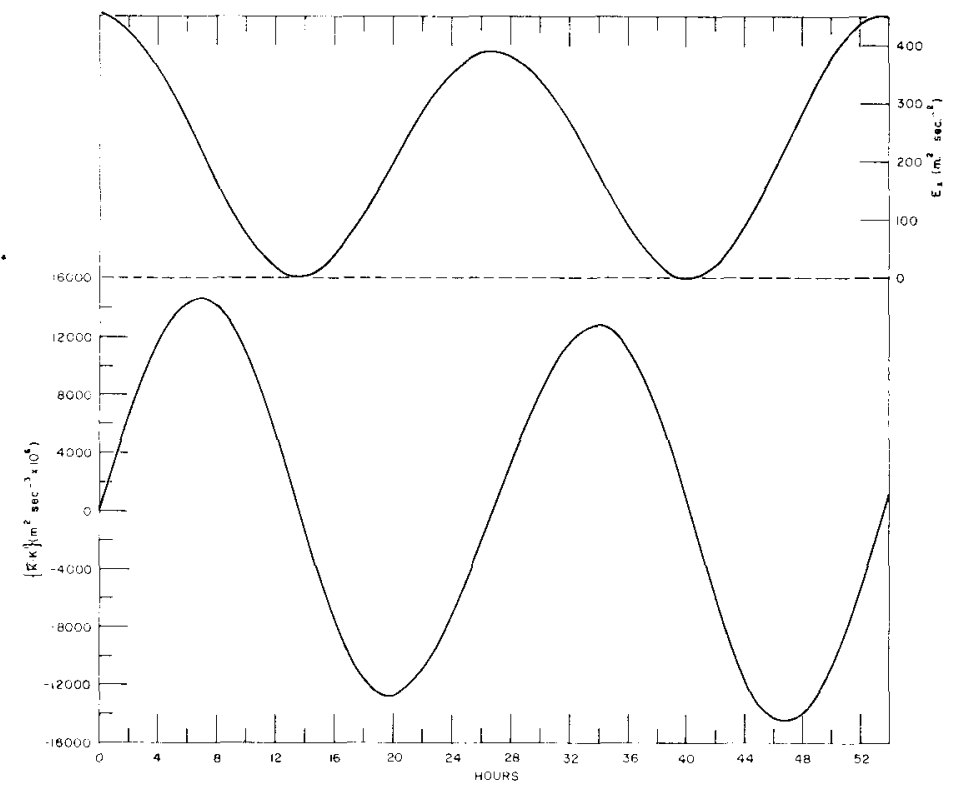

Figtre 17. Case 2. Zonal kinetic energy as a function of time over one period (upper curve, right scale). The energy conversion between zonal and eddy kinetic energy as a function of time over one period (lower curve, left scale).

ment with the energy conversion curve we find a decrease in the zonal kinetic energy up to 15 hours, when it increases slightly, and then a decrease again before it finally starts to increase to complete the cycle after 44 hours.

We can compute the amount of unavailable zonal kinetic energy from the formula (6.17). With $B_{0}=30$ m.sec. ${ }^{-1}$ and $C_{0}=-15 \mathrm{~m} . \mathrm{sec}^{-1}$ we find $M_{0}=15 \mathrm{~m}$. sec. $^{-1}$ The minimum zonal kinetic energy is therefore $E_{z, m i n}=$ $140.6 \mathrm{~m}^{2} \mathrm{sec}^{-2}$ It is interesting to note that this is equal to the actual minimum on the curve for $E_{z}$, indicating that all the available kinetic energy in the zonal flow actually is being released to the perturbations. It also indicates that very large amounts of kinetic energy 
may be released to the perturbations if we specily the initial zonal wind profile in such a way that the momentum is small. In order to test this we computed a second case. Case 2: In this case we have chosen $B=-C=30 \mathrm{~m}$. sec. $^{-1}$, which makes the zonal momentum $M_{0}=0$. The unavailable kinetic energy vanishes therefore in this case, and the total amount of kinetic energy is at the same time larger. In this admittedly extreme case we have again a maximum of $60 \mathrm{~m}$. sec. ${ }^{-1}$ in the center of the channel in the initial, zonal wind profile with easterlies to the north and south of $33 \mathrm{~m}$. sec.-1 (fig. 16). The period in the forecasts turns out to be about 54 hours in this case. Very violent fluctuations take place in the zonal winds at the different latitudes in this case. In the middle of the period we find easterlies in the center of the channel of $55 \mathrm{~m} . \mathrm{sec}^{-1}$, while the westerlies in the northern and southern portions, at the same time, have increased in strength to more than $30 \mathrm{m.sec}{ }^{-1}$ It can be seen on figure 16 that the forecast passes through a state between 13 and 14 hours in which there is no zonal wind, a situation which is found again between 40 and 41 hours.

Figure 17 (corresponding to fig. 15 in case 1) shows the time variation of the zonal kinetic energy and the energy conversion between zonal and eddy kinctic energy. We find as expected that the zonal kinetic energy decreases to zero between 13 and 14 hours and again between 40 and 41 hours. The energy conversion is naturally zero at the same time after having been positive, since all the kinetic energy has been converted to eddy kinetic energy. The secondary maximum in the zonal kinetic energy in the middle of the period where the energy conversion again is zero corresponds to the maximum intensity of the double jet stream seen in figure 16 at 27 hours.

Case 3: The initial zonal wind profile for this case was similar to case 1 in the sense that $B$ and $C$ initially had the same values $\left(B_{0}=30\right.$ m.sec. ${ }^{-1}, C_{0}=-15$ m.sec. ${ }^{-1}$ ), but the dimensions of the region were increased. The width of the channel was taken to be $10,000 \mathrm{~km}$., equal to the distance between pole and equator on the earth. The wavelength in the zonal direction was also taken to be $10,000 \mathrm{~km}$., making the initial flow stable according to the linear theory (see figs. 2 and 3 ). The initial perturbation was again prescribed by setting $E_{1}=25 \mathrm{~m}$.sec. $^{-1}$ initially, while all the other amplitudes were zero initially.

Some results from this forecast are shown in figures 18 and 19. The first (fig. 18) shows a record of the strength of the zonal wind in the center of the channel and at a distance $0.2 D$ from the wall. The zonal wind in the center shows a regular variation between $60 \mathrm{~m}$.sec. ${ }^{-1}$ and $32.5 \mathrm{~m}_{\text {.sec. }}{ }^{-1}$ with a period of slightly more than 6 days, while the variation closer to the wall is between -6 m.sec. ${ }^{-1}$ and +9 m.sec. ${ }^{-1}$ with the same period. We find again a tendency to divide the initial single jet maximum into two maxima, because the westerly wind close to the wall is at a maximum at the same time as the zonal wind

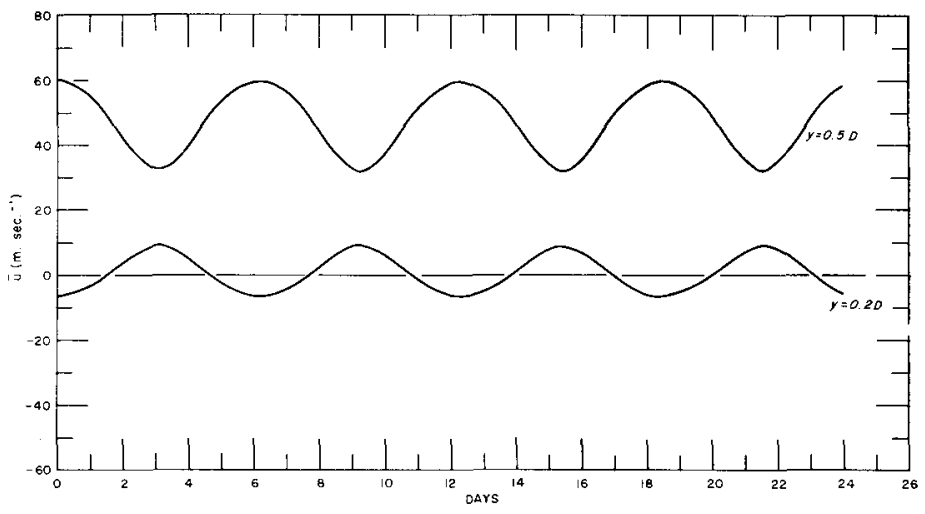

Figure 18.-Case 3. The zonal velocity in the center of the channel and at a distance of $0.2 \mathrm{D}$ from the wall as a function of time. Parameters $B_{o}=30 \mathrm{~m}$. sec. ${ }^{-1}, C_{o}=15 \mathrm{~m}$. sec. ${ }^{-1}, E_{10}=$ $F_{10}=F_{3 j}=0, \beta=\beta_{45}$, width $10,000 \mathrm{~km}$, , zonal wavelength 10,000 $\mathrm{km}$.

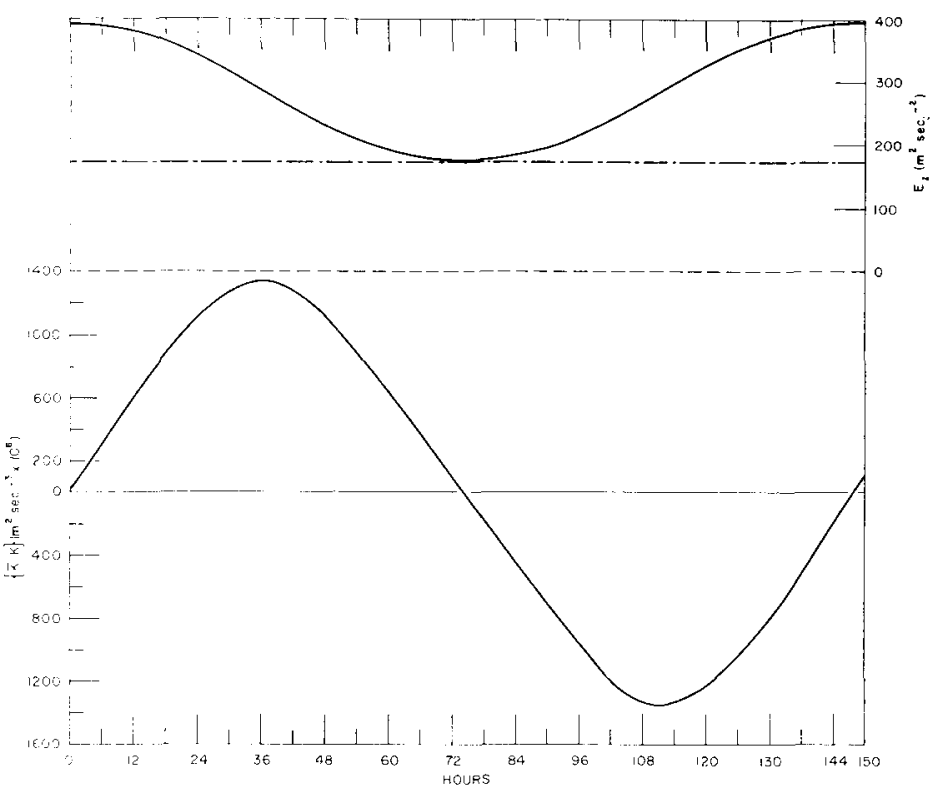

Figtre 19.-Case 3. The zonal kinetic energy as a function of time over one period (upper curve, right scale). The energy conversion between zonal and eddy kinetic energy as a function of time over one period (lower curve, left scale).

is at a minimum in the center of the channel. The energy conversion between zonal and eddy kinetic energy is plotted in figure 19 as a function of time together with the time variation of the zonal kinetic energy itself over one period (about 148 hours). 'The two curves have this time a very regular variation, where the zonal kinetic energy goes to a minimum equal to the value estimated from equation (6.17) in the middle of the period.

Case 4: The initial specifications for this case were $B_{0}=30 \mathrm{~m}$.sec. ${ }^{-1}, C_{0}=-30 \mathrm{~m} . \mathrm{sec}^{-1}$, the width of the channel $10,000 \mathrm{~km}$., and the zonal wavelength $10,000 \mathrm{~km}$. We have therefore again chosen a situation where the total zonal momentum vanishes. The variation of the zonal wind in the center of the channel and at a distance of $0.2 D$ from the wall is shown in figure 20 as a function of time over a 24-day period. The zonal wind in the center 


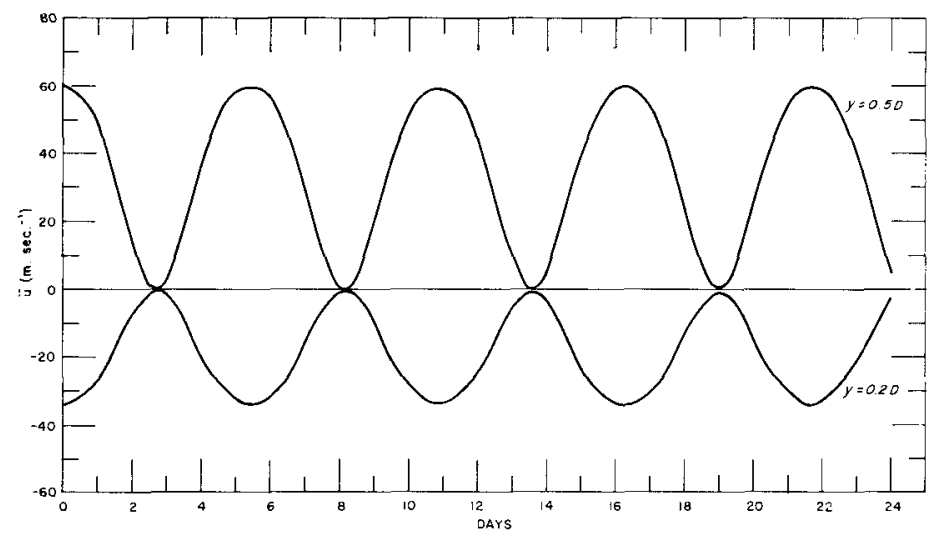

Figure 20.-Case 4. The zonal velocity in the center of the channel and at a distance of $0.2 \mathrm{D}$ from the wall as a function of time. Parameters as in case 3 (fig. 18) except $C_{0}=-30 \mathrm{~m}$. sec. ${ }^{-1}$.

varies between $60 \mathrm{~m} . \mathrm{sec}^{-1}$ and $0 \mathrm{~m} . \mathrm{sec} .^{-1}$ with a period of $5 \frac{1}{2}$ days, while the zonal wind at $0.2 D$ varies between -33 m.sec. ${ }^{-1}$ and 0 m.sec. ${ }^{-1}$. Notice, that no zonal wind exists at $2^{3 / 4}$ days and at intervals of $5 \frac{1}{2}$ days thereafter. This picture is consistent with the curves in figure 21 where we notice that the kinetic energy of the zonal flow goes to zero in the middle of the period.

The characteristic period of 5-6 days which has been found in these integrations agrees with the typical time scale found by Thompson [15] in his theory of longperiod variations in barotropic flow. A further agreement is found between the results obtained here and those reported by Charney [4] from a single extended integration of the barotropic vorticity equation and by Baer [1].

Some support is also found in analysis of atmospheric data for periodicities of this order of magnitude. Reference is made to the observational studies by Mintz and Kao [10] who found a period of 3-5 days in the convergence of the meridional transport of momentum at certain latitudes during January 1949. The study by Duggan [6] of the meridional convergence of momentum also shows periodicities of this order of magnitude.

The results of the non-linear integrations can be used to obtain information about how soon the higher-order effects become important. One may, for example, obtain this information from curves giving the energy conversion from zonal kinetic energy to eddy kinetic energy as a function of time in the non-linear computation and estimated from the linear theory including terms of different orders.

Substituting the expression (3.19) for $\bar{u}(y, t)$ and (3.18) for $-\partial\left(\overrightarrow{u^{\prime} v^{\prime}}\right) / \partial y$ in the formula $(5.12)$ for the energy conversion we find first that

$$
\begin{aligned}
& \left\{\bar{K} \cdot K^{\prime}\right\}=4 \mu \lambda^{2} N^{*} \sinh (2 \mu S t) \cdot\left[\frac{1}{D} \int_{0}^{D} \bar{u}(y, 0) M(y) d y\right] \\
& -\frac{8 \mu \lambda^{4} N^{* 2}}{S}(\cosh (2 \mu S t)-1) \sinh (2 \mu S t) \cdot\left[\frac{1}{D} \int_{0}^{D} M^{2}(y) d y\right] .
\end{aligned}
$$

The first term on the right hand side of (6.19) gives the

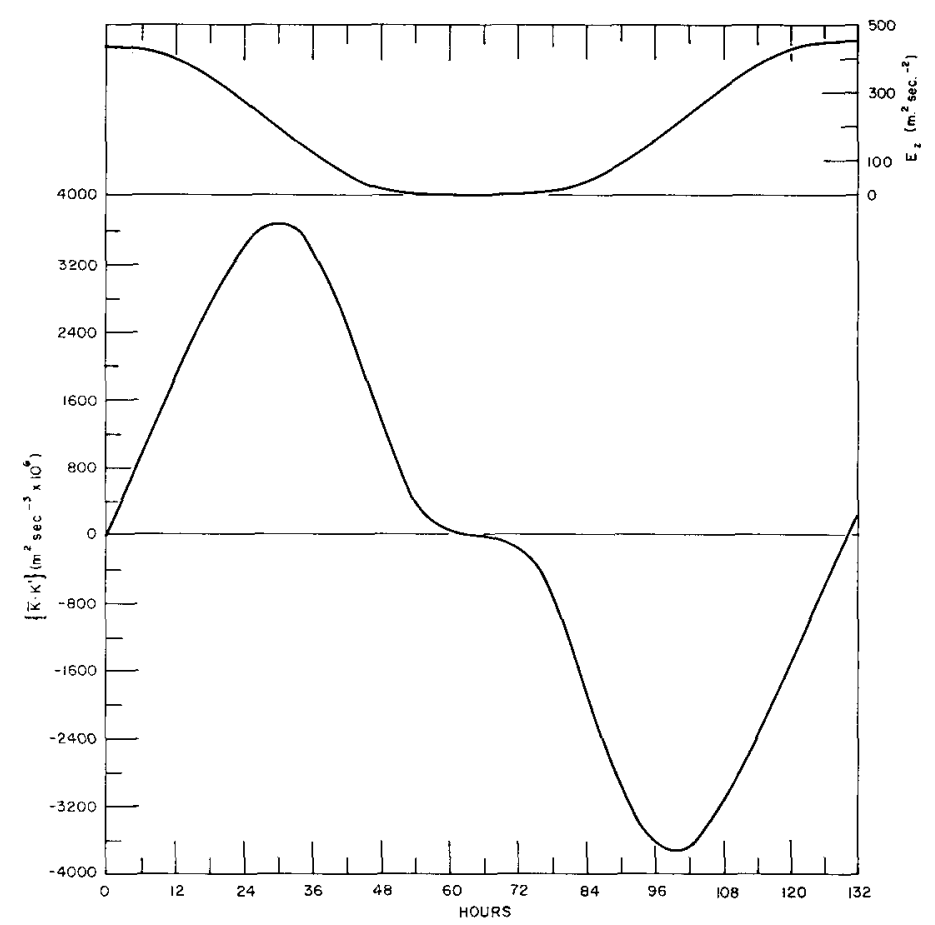

Figure 21. - Case 4. The zonal kinetic energy as a function of time over one period (upper curve, right scale). The energy conversion between zonal and eddy kinetic energy as a function of time over one period (lower curve, left scale).

contribution which depends upon the initial profile $u$ $(y, 0)$, while the second term is independent of the initial profile. Since the meridional distribution of the convergence of momentum transport, expressed by the function $M(y)$, is a second-order effect, we may say that the first term in $\left\{\bar{K} \cdot K^{\prime}\right\}$ is of second order, while the last term is a fourth-order effect.

When the expressions (2.9) for $\bar{u}(y, 0)$ and (3.15) for $M(y)$ are introduced in (6.19) we obtain after evaluation of the integrals

$$
\begin{aligned}
\left\{\bar{K} \cdot K^{\prime}\right\}= & 2 \mu \lambda^{2} N^{*} B \sinh (2 \mu S t) \\
& -\frac{8 \mu \lambda^{4} N^{* 2}}{S} \sinh (2 \mu S t) \cdot(\cosh (2 \mu S t)-1) .
\end{aligned}
$$

The following case was selected for comparison. The zonal wavelength was chosen to be $5,000 \mathrm{~km}$., the maximum meridional wavelength to be $6,000 \mathrm{~km}$., $\beta=16 \times$ $10^{-12} \mathrm{~m}^{-1}$ sec. $^{-1}, B=30 \mathrm{~m}$. sec. ${ }^{-1}$, and $q^{2}=0$. The curve without marks in figure 22 gives the energy conversion in the non-linear computation as a function of time. The energy conversion increases to a maximum, which is reached after approximately 44 hours. The curve in figure 22 marked with circles is the energy conversion computed from (6.20) including, however, only the first term. By a comparison of the two curves we find that the curve including the second-order effects gives a good estimate of the energy conversion up to approximately 24 hours, although the estimate is slightly too high. When the fourth-order effects are included in the evaluation of (6.20), 


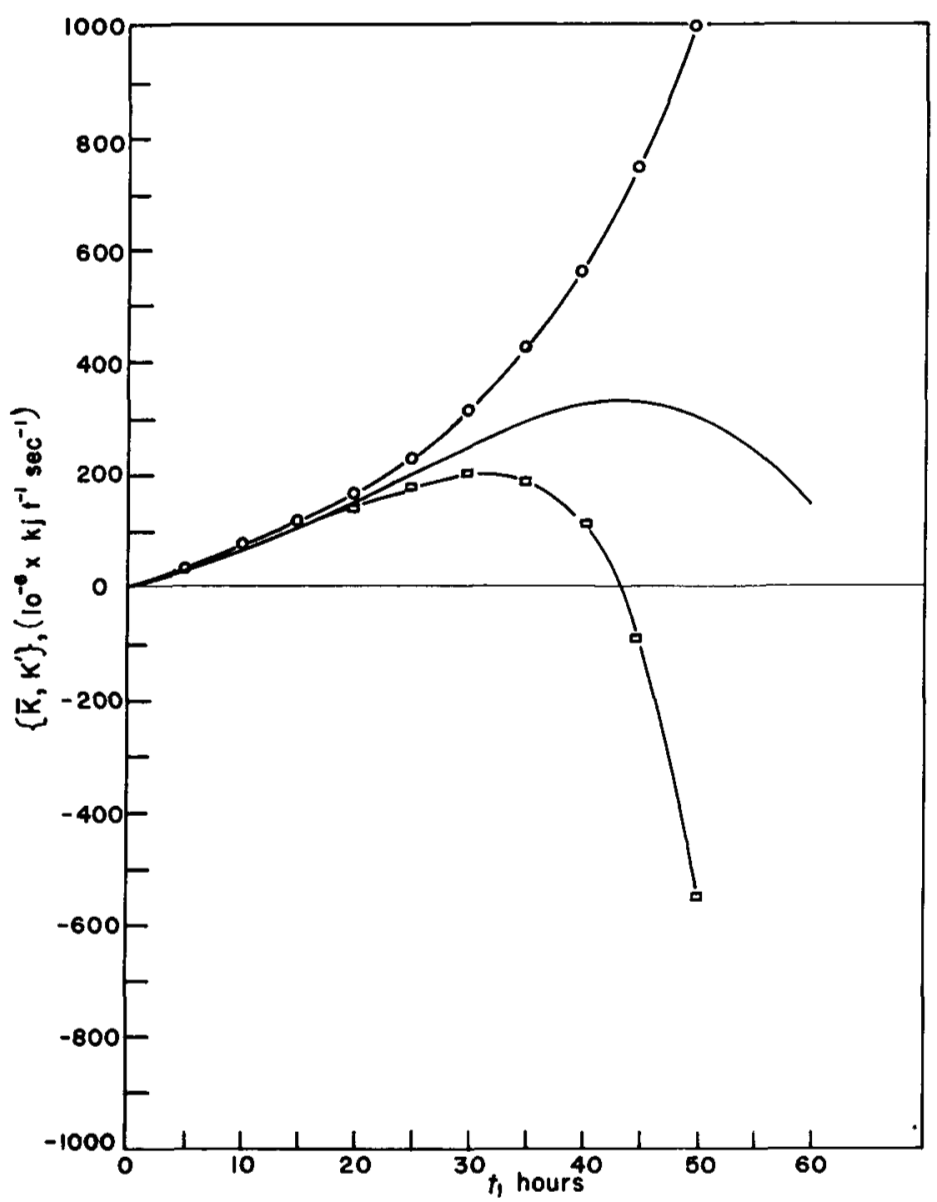

Figure 22.- -The curve without marks gives the energy conversion as a function of time in a non-linear calculation with the following parameters: zonal wavelength $5,000 \mathrm{~km}$., maximum meridional wavelength $6,000 \mathrm{~km} ., \beta=16 \times 10^{-12} \mathrm{~m}^{-1} \mathrm{sec}^{-1}, B=30 \mathrm{~m}$. sec. ${ }^{-1}$, and $q^{2}=0$. The curve marked with circles gives the energy conversion computed from the linear solution using the same parameters and including second-order effects, while the curve marked with squares also includes fourth-order effects.

we obtain the curve in figure 22 marked with squares. This curve gives a very good estimate for a little longer period than the second-order curve, but then rapidly shows an underestimate. The main result of the inclusion of the fourth-order term in (6.20) is, however, that this term actually predicts that the energy conversion will reach a maximum. The main conclusion from figure 22 is that the higher-order effects become important after about 1 day.

\section{SUMMARY}

The first sections of this paper contain a stability investigation of the divergent, one-parameter model. The main conclusion is that the introduction of a divergence term into the model tends to reduce the instabilities present in the non-divergent model. The second-order effects of the disturbances on the profile of the zonal wind are investigated. It is found that the unstable barotropic disturbances tend to cause a decrease of the zonal winds in the center of the channel and an increase of the mean zonal winds to the north and south.
A simple non-linear barotropic model containing only a few wave components is investigated in the next sections through integrations over extended time periods. The changes in the profile of the zonal wind, the energy conversion between kinetic energy of the mean flow and the eddies, and the time periodicities of the flow are investigated. Fluctuations of the order of 2 days are found in initially unstable flow, while the large-scale stable flow pattern shows periods of the order of 6 days.

\section{REFERENCES}

1. F. Baer, "The Extended Numerical Integration of a Simple Barotropic Model, Part II," Technical Report No. 3, National Science Foundation (NSF-G 2159), 1958.

2. B. W. Boville, "A Dynamical Study of the 1958-59 Stratospheric Polar Vortex," Scientific Report No. 9, McGill University, Contract No. AF19(604)-3865, 1961, 134 pp.

3. K. Bryan, "A Numerical Investigation of Certain Features of the General Circulation," Tellus, vol. 11, No. 2, May 1959, pp. $163-174$.

4. J. Charney, "On the Theory of the General Circulation of the Atmosphere," pp. 178-193 of The Atmosphere and the Sea in Motion, Rossby Memorial Volume, Rockefeller Institute Press, New York, 1959.

5. G. P. Cressman, "Barotropic Divergence and Very Long Atmospheric Waves," Monthly Weather Review, vol. 86, No. 8, Aug. 1958, pp. 293-297.

6. F. F. Duggan, "A Study of the Meridional Convergence of Angular Momentum at 500 mbs. in Selected Latitude Belts," Thesis, U.S. Naval Postgraduate School, 1960. 16 pp.

7. E. Eliasen, "Numerical Solutions of the Perturbation Equation for Linear Flow," Tellus, vol. 6, No. 2, May 1954, pp. 183-192.

8. H. Kuo, "Dynamic Instability of Two-Dimensional NonDivergent Flow in a Barotropic Atmosphere," Journal of Meteorology, vol. 6, No. 2, Apr. 1949, pp. 105-122.

9. E. N. Lorenz, "Maximum Simplification of the Iynamic Equations," Tellus, vol. 12, No. 3, Aug. 1960, pp. 243-254.

10. Y. Mintz and S-K. Kao, "A Zonal-Index Tendency Equation and Its Application to Forecasts of the Zonal Index," Journal of Meteorology, vol. 9, No. 2, Apr. 1952, pp. 87-92.

11. F. W. Murray, "Dynamic Stability in the Stratosphere," Journal of Geophysical Research, vol. 65, No. 10, Oct. 1960, pp. 3273-3305.

12. N. A. Phillips, "General Circulation of the Atmosphere: A Numerical Experiment," Quarterly Journal of the Royal Meteorological Society, vol. 82, No. 352, Apr. 1956, pp. 123-164.

13. G. W. Platzman and F. Baer, "The Extended Numerical Integration of a Simple Barotropic Model, Part I," Technical Report, No. 1, National Science Foundation (NSF-G 2159), 1958.

14. B. Saltzman, "Numerical Solution for an Idealized Barotropic Flow, Studies of the Atmospheric General Circulation III," Final Report, Massachusetts Institute of Technology, Contract No. AF 19(604)-2242, 1959, pp. 132-147.

15. P. D. Thompson, "A Heuristic Theory of Large-Scale Tur bulence and Long-Period Velocity Variations in Barotropic Flow," Tellus, vol. 9, No. 1, Feb. 1957, pp. 69-91.

16. W. Tollmien, "Utber die Entstehung der Turbulenz, I," Mitteilung, Nachrichten Gesellschaft Wissenschaften, Göttingen (Neue Folge), vol. 1, 1929, pp. 20-44.

17. A. Wiin-Nielsen, "On Barotropic and Baroclinic Models, with Special Emphasis on Ultra-Long Waves," Monthly Weather Review, vol. 87, No. 5, May 1959, pp. 171-183. 